\title{
Entanglement negativity and minimal entanglement wedge cross sections in holographic theories
}

\author{
Jonah Kudler-Flam* and Shinsei Ryu ${ }^{\dagger}$ \\ James Franck Institute and Kadanoff Center for Theoretical Physics, \\ University of Chicago, Illinois 60637, USA
}

(Received 31 October 2018; published 28 May 2019; corrected 3 June 2019)

\begin{abstract}
We calculate logarithmic negativity, a quantum entanglement measure for mixed quantum states, in quantum error-correcting codes and find it to equal the minimal cross sectional area of the entanglement wedge in holographic codes with a quantum correction term equal to the logarithmic negativity between the bulk degrees of freedom on either side of the entanglement wedge cross section. This leads us to conjecture a holographic dual for logarithmic negativity that is related to the area of a cosmic brane with tension in the entanglement wedge plus a quantum correction term. This is closely related to (though distinct from) the holographic proposal for entanglement of purification. We check this relation for various configurations of subregions in $\mathrm{AdS}_{3} / \mathrm{CFT}_{2}$. These are disjoint intervals at zero temperature, as well as a single interval and adjacent intervals at finite temperature. We also find this prescription to effectively characterize the thermofield double state. We discuss how a deformation of a spherical entangling region complicates calculations and speculate how to generalize to a covariant description.
\end{abstract}

DOI: $10.1103 /$ PhysRevD.99.106014

\section{INTRODUCTION}

Holographic duality (the AdS/CFT correspondence) [1-3] has made a dramatic impact on how we understand theories of quantum gravity and strongly coupled conformal field theories. One of the recent explorations in this context is a connection to quantum information theory, aiming to uncover the mechanism of holographic duality and quantum gravity [4-7]. In particular, it has been proposed that the duality can be interpreted as a quantum error-correcting code [8-10]. This surprising connection has been able to shed light on mysterious parts of holographic duality. For example, it helps to explain the holographic formula of entanglement entropy, which equates the von Neumann entropy of the boundary conformal field theory (CFT) to the geometry of the bulk anti-de Sitter (AdS) [11,12]:

$$
S\left(\rho_{A}\right)=\frac{\operatorname{Area}\left(\mathcal{L}_{A}\right)}{4 G_{N}}+S_{\text {bulk }}
$$

Here, $S\left(\rho_{A}\right)$ is the von Neumann entropy of the subspace $A$ in the boundary CFT, and $\mathcal{L}_{A}$ is the extremal surface in the bulk

\footnotetext{
jikudlerflam@uchicago.edu

ryuu@uchicago.edu
}

Published by the American Physical Society under the terms of the Creative Commons Attribution 4.0 International license. Further distribution of this work must maintain attribution to the author(s) and the published article's title, journal citation, and DOI. Funded by SCOAP. homologous to $A ; G_{N}$ is the bulk Newton constant. $S_{\text {bulk }}$ is the bulk entanglement entropy of the corresponding entanglement wedge, the quantum correction term $[13,14]$. This formula was shown to hold in the case of "holographic states" made of perfect tensors [10] and in random tensor networks [15]. It was later proven more generally for quantum error-correcting codes [8].

For mixed quantum states, the von Neumann entropy is not a proper measure for the quantum correlation; it captures classical (thermal) correlations as well as purely quantum ones. The (logarithmic) entanglement negativity is a measure of quantum entanglement, which can be applied to mixed states. In the quantum field theory context, for example, it has been computed and discussed for $(1+1)$ d CFTs and $(2+1) \mathrm{d}$ topological quantum field theories [16-31].

In this paper, we will make a comparison, in the holographic context, between the entanglement negativity and the minimal cross sectional area of entanglement wedges. The entanglement wedge has been proposed as a natural bulk region corresponding to a given boundary region [32-34] and has proven to be an important concept, distinct from the causal wedge, when discussing bulk reconstruction [9,10,33-36]. In addition, it was recently proposed that minimal entanglement wedge cross sections are a measure of the entanglement of purification (EoP) [37-45]. We will discuss the distinctions between the bulk objects proposed as duals to the logarithmic negativity and EoP. It is also worth mentioning that there is a proposal for holographic negativity that relates certain combinations of bulk geodesics to the negativity in the boundary CFT [46-50]. 
First, we consider the logarithmic negativity in generic quantum error-correcting codes. With this formalism, we study negativity and the entanglement wedge cross section in holographic quantum error-correcting codes-they are toy models of holographic duality. There, as we will see, the logarithmic negativity is equivalent to the cross sectional area of entanglement wedges with a bulk quantum correction term. We explicitly show this for setups where we bipartition the (boundary) system at finite temperature as well as arbitrary tripartitions.

With motivations from quantum error-correcting codes, we next conjecture a general bulk object that computes the logarithmic negativity. While for general entangling surface geometries this is difficult to compute due to the backreaction of the cosmic branes that we will introduce, the calculation is greatly simplified for ball-shaped subregions. In these symmetric setups, the backreaction is accounted for by an overall constant to the area of the entanglement wedge cross section. We then conjecture that the logarithmic negativity, $\mathcal{E}$, in holographic CFTs is given by

$$
\mathcal{E}=\mathcal{X}_{d} \frac{E_{W}}{4 G_{N}}+\mathcal{E}_{\text {bulk }}
$$

where $E_{W}$ is the minimal cross sectional area of the entanglement wedge associated with the boundary region of interest and $\mathcal{X}_{d}$ is a constant which depends on the dimension of the spacetime. $\mathcal{E}_{\text {bulk }}$ is the quantum correction term corresponding to the logarithmic negativity between the bulk fields on either side of the cross section. In $\mathrm{AdS}_{3} / \mathrm{CFT}_{2}$ (where $\mathcal{X}_{d}=3 / 2$ ), we find that the entanglement wedge cross section formula reproduces many known properties of the logarithmic negativity in $(1+1) \mathrm{d}$ (holographic) CFTs.

For the rest of the Introduction, we briefly recall the definitions of the key concepts in this paper: the (logarithmic) entanglement negativity, the entanglement wedge, and holographic error-correcting codes.

\section{A. Entanglement negativity}

For bipartite pure states, the von Neumann entropy of the reduced density matrix effectively encapsulates the quantum correlations between subsystems. However, when working with mixed states, the von Neumann entropy is not a proper entanglement measure; for example, the von Neumann entropy additionally counts the classical correlations. In particular, in thermal systems without quantum correlations, this will just be the regular thermal entropy.

The (logarithmic) negativity was proposed as a computable measure of quantum entanglement for mixed states [51-55]. The negativity is a measure derived from the positive partial transpose criterion for the separability of mixed states [51] and is defined/computed by taking the trace norm of the partial transpose of the density matrix; for the Hilbert space $\mathcal{H}_{A} \otimes \mathcal{H}_{B}$, the partial transpose of the density matrix $\rho$ is defined in terms of its matrix elements as

$$
\left\langle i_{A}, j_{B}\left|\rho^{T_{A}}\right| k_{A}, l_{B}\right\rangle=\left\langle k_{A}, j_{B}|\rho| i_{A}, l_{B}\right\rangle,
$$

where $\left\{\left|i_{A / B}\right\rangle\right\}$ represent the orthonomal basis for $\mathcal{H}_{A / B}$. The entanglement negativity and logarithmic negativity are defined as

$$
\begin{aligned}
\mathcal{N}(\rho) & :=\frac{1}{2}\left(\left|\rho^{T_{A}}\right|_{1}-1\right), \\
\mathcal{E}(\rho) & :=\ln \left|\rho^{T_{A}}\right|_{1},
\end{aligned}
$$

where $|A|_{1}:=\operatorname{Tr} \sqrt{A A^{\dagger}}$. In this paper, we will be mainly concerned with the logarithmic negativity (and hence by entanglement negativity, we refer to $\mathcal{E}$ unless stated otherwise).

\section{B. Entanglement wedge}

The entanglement wedge is the bulk region corresponding to the reduced density matrix on the boundary. In this paper, we are only concerned with entanglement of the CFT on the boundary on a fixed time slice, corresponding to a Cauchy slice of AdS in the bulk. Relevant generalizations of entanglement entropy to time-dependent situations have been studied in Refs. [56-58]. Given a Cauchy slice, $\Xi$, and a subset of the conformal boundary, $A \subset \partial \Xi$, the relevant surface, $\gamma_{A}$, is the codimension-2 extremal surface homologous to $A, \partial A=\partial \gamma_{A}$. The corresponding entanglement wedge of $A$ is the codimension-1 surface in $\Xi$ of which the boundary is $\gamma_{A} \cup A$.

We are interested in the minimal cross sectional area of the entanglement wedge. This picture is intuitive when the bulk does not contain horizons. However, when there is a black hole in the bulk, the entanglement wedge cross section can become disconnected (Fig. 1).

\section{Holographic codes}

A series of concrete and exactly solvable toy models of holography, holographic codes, were proposed in Ref. [10]. Leveraging the fact that the AdS/CFT correspondence shares central properties with quantum error-correcting
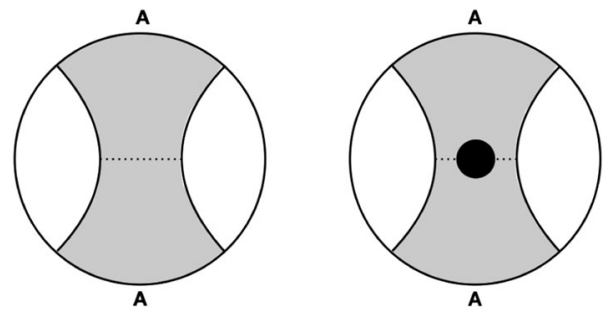

FIG. 1. The gray bulk region is the entanglement wedge of boundary subregion $A$. The dotted line represents the minimal entanglement wedge cross section. The figure on the right displays a black hole. The cross section then becomes disconnected, containing pieces on either side of the black hole but not including any of the horizon. 


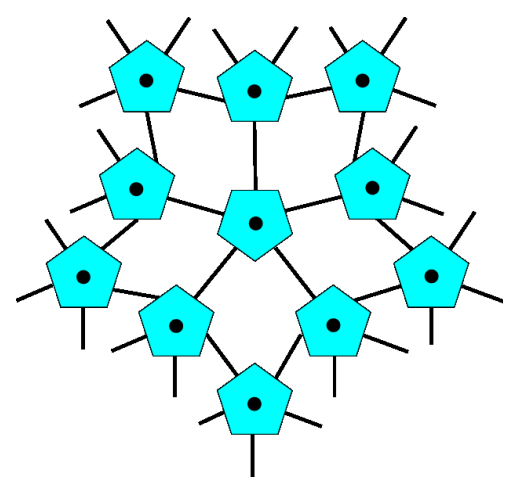

FIG. 2. The holographic pentagon code introduced in Ref. [10]. Each perfect tensor, represented by a pentagon, has six indices, with one free bulk index (represented by dots).

codes, the authors studied a tensor network description of a quantum-error-correcting code living on a given twodimensional time slice. This code acts as an isometric map from the bulk Hilbert space to the boundary Hilbert space. This "holographic code" is composed of perfect tensors which are tensors such that any partition of indices into $\mathcal{H}_{A}$ and $\mathcal{H}_{B}$ induces an isometry $T$ from $\mathcal{H}_{A}$ to $\mathcal{H}_{B}$, given that $\left|\mathcal{H}_{A}\right| \leq\left|\mathcal{H}_{B}\right|$. An example of a holographic code model, the holographic pentagon code, is depicted in Fig. 2. For a complete discussion of such codes, see Ref. [10].

\section{ENTANGLEMENT WEDGE AND NEGATIVITY IN QUANTUM ERROR-CORRECTING CODES}

In this section, we will calculate the logarithmic negativity in generic quantum error-correcting codes. We will later use this technology to gain geometrical insight into logarithmic negativity in holographic codes. Following the structure of Ref. [8], we warm up by starting with simple erasure-correcting code models for holography and then continue to more general error-correcting codes.

\section{A. Conventional QEC}

We work with a total Hilbert space $\mathcal{H}$ endowed with the tensor product structure $\mathcal{H}=\mathcal{H}_{A} \otimes \mathcal{H}_{\bar{A}} ; \mathcal{H}_{A}=\left(\mathcal{H}_{A_{1}} \otimes\right.$ $\left.\mathcal{H}_{A_{2}}\right) \oplus \mathcal{H}_{A_{3}}$. The logical state is then encoded in the state subspace such that there exists a unitary $U_{A}$ such that

$$
|\tilde{i}\rangle=U_{A}\left(|i\rangle_{A_{1}} \otimes|\chi\rangle_{A_{2}, \bar{A}}\right), \quad|\chi\rangle_{A_{2}, \bar{A}} \in \mathcal{H}_{A_{2}, \bar{A}} .
$$

This implies a code that corrects for the erasure of $\bar{A}$. Here, the state $|\chi\rangle_{A_{2}, \bar{A}}$ is our entanglement resource for quantum error correction.

The three-qutrit code is the simplest example of conventional quantum error correction that displays holographic properties. It consists of three physical qutrits, each with states $|0\rangle,|1\rangle$, and $|2\rangle$, that encode a single logical qutrit $|\tilde{i}\rangle$ as follows:

$$
\begin{aligned}
& |\tilde{0}\rangle=\frac{1}{\sqrt{3}}(|000\rangle+|111\rangle+|222\rangle), \\
& |\tilde{1}\rangle=\frac{1}{\sqrt{3}}(|012\rangle+|120\rangle+|201\rangle), \\
& |\tilde{2}\rangle=\frac{1}{\sqrt{3}}(|021\rangle+|102\rangle+|210\rangle) .
\end{aligned}
$$

This code can correct for the erasure of any single physical qutrit because there exists a unitary operator, $U_{A_{1} A_{2}}$, such that

$U_{A_{1} A_{2}}^{\dagger}|\tilde{i}\rangle=|i\rangle_{A_{1}}|\chi\rangle_{A_{2} \bar{A}}, \quad|\chi\rangle \equiv \frac{1}{\sqrt{3}}(|00\rangle+|11\rangle+|22\rangle)$,

where $A_{1}, A_{2}$, and $\bar{A}$ correspond to the three qutrits. See Ref. [9] for the explicit $U_{A}$. Because of the symmetry of the code, there also exist analogous unitary operators $U_{A_{2} \bar{A}}$ and $U_{A_{1} \bar{A}}$. In Refs. [8,9], this simple code is shown to contain analogs of many important aspects of holography: black holes, effective field theory, radial commutativity, subregion duality, and the holographic formula of entanglement entropy (the Ryu-Takayanagi (RT) formula).

We will only replicate the argument for the RT formula here because the other properties do not directly apply to the calculation and interpretation of the negativity of this code. Consider an arbitrary mixed state of a conventional quantum error-correcting code

$$
\tilde{\rho}=U_{A_{1} A_{2}}\left(\rho_{A_{1}} \otimes|\chi\rangle\left\langle\left.\chi\right|_{A_{2}, \bar{A}}\right) U_{A_{1} A_{2}}^{\dagger},\right.
$$

where $\rho_{A_{1}}$ is an arbitrary mixed input state. Defining $\chi_{A_{2}} \equiv \operatorname{Tr}_{\bar{A}}|\chi\rangle\left\langle\left.\chi\right|_{A_{2}, \bar{A}}\right.$, the von Neumann entropies for the reduced density matrices $\tilde{\rho}_{A}=\operatorname{Tr}_{\bar{A}} \tilde{\rho}$ and $\tilde{\rho}_{\bar{A}}=\operatorname{Tr}_{A} \tilde{\rho}$ are

$$
S\left(\tilde{\rho}_{A}\right)=S\left(\chi_{A_{2}}\right)+S(\tilde{\rho}), \quad S\left(\tilde{\rho}_{\bar{A}}\right)=S\left(\chi_{A_{2}}\right) .
$$

By identifying $S\left(\chi_{A_{2}}\right) I_{\text {code }}$ as the "area operator," $\mathcal{L}$,

$$
\langle\mathcal{L}\rangle=S\left(\chi_{A_{2}}\right)=-\sum_{a} p_{a} \ln p_{a}
$$

an RT-like formula for error-correcting codes is obtained. $\mathcal{L}$ can be thought of as an area because it contributes equally to $A$ and $\bar{A}$. Furthermore, if one works with tensor networks, $\mathcal{L}$ originates from $|\chi\rangle$, which make up the Hilbert space of the contracted legs of the network. Though this initial formulation of error-correcting codes displays certain aspects of holography, it is not entirely satisfactory. This is partially due to the entanglement entropy not being symmetric. Only for system $A$ is there a bulk entropy term. In the next section, we expand to more general errorcorrecting codes so that both entropies contain bulk entropy terms, as we expect they should. Another motivation for 
this generalization is that we will be able to apply our results to the holographic codes introduced in Ref. [10]. The ability to use holographic codes will be crucial in our analysis of negativity in tripartitions.

Before discussing more generic holographic code models, let us consider the negativity of the conventional quantum error correction (QEC) model. In order to take the partial transpose with respect to $A$ or $\bar{A}$, we need to perform a Schmidt decomposition of $|\chi\rangle$ :

$$
|\chi\rangle=\sum_{a} \sqrt{p_{a}}|a\rangle_{A_{2}} \otimes|a\rangle_{\bar{A}}
$$

$|\chi\rangle$ is maximally entangled when

$$
p_{a}=\frac{1}{|\tilde{A}|}, \quad|\tilde{A}|=\min \left(\left|A_{2}\right|,|\bar{A}|\right) .
$$

Taking the partial transpose with respect to $\bar{A}$ [59],

$$
\begin{aligned}
\tilde{\rho}^{T_{\bar{A}}} & =\sum_{a, b} \sqrt{p_{a} p_{b}} U_{A_{1} A_{2}}\left(\rho_{A_{1}} \otimes|a\rangle\left\langle\left. b\right|_{A_{2}} \otimes \mid b\right\rangle\left\langle\left. a\right|_{\bar{A}}\right) U_{A_{1} A_{2}}^{\dagger},\right. \\
\tilde{\rho}^{T_{\bar{A}}^{\dagger}} \tilde{\rho}^{T_{\bar{A}}} & =\sum_{a, b} p_{a} p_{b} U_{A_{1} A_{2}}\left(\rho_{A_{1}}^{2} \otimes|a\rangle\left\langle\left. a\right|_{A_{2}} \otimes \mid b\right\rangle\left\langle\left. b\right|_{\bar{A}}\right) U_{A_{1} A_{2}}^{\dagger},\right. \\
\left|\tilde{\rho}^{T_{\bar{A}}}\right|_{1} & =\left(\sum_{a} \sqrt{p_{a}}\right)^{2} .
\end{aligned}
$$

(See Fig. 3 for graphical representations of these objects, when $|\chi\rangle$ is maximally entangled.) We obtain the entanglement negativity $\mathcal{N}$ and logarithmic negativity $\mathcal{E}$,

$$
\begin{gathered}
\mathcal{N}(\tilde{\rho})=\frac{\left(\sum_{a} \sqrt{p_{a}}\right)^{2}-1}{2}, \\
\mathcal{E}(\tilde{\rho})=\ln \left(\sum_{a} \sqrt{p_{a}}\right)^{2}=S_{1 / 2}\left(\chi_{A_{2}}\right),
\end{gathered}
$$

where $S_{1 / 2}$ is the Rényi entropy with Rényi index $1 / 2$. So, the negativity is equal to the expectation value of the area operator $\langle\mathcal{L}\rangle$ when $\chi_{A_{2}}$ is maximally mixed:

$$
\mathcal{E}(\tilde{\rho})=\langle\mathcal{L}\rangle=\ln (|\tilde{A}|) .
$$

For tensor networks, because the spectrum of the entanglement Hamiltonian is flat, $\chi_{A_{2}}$ is maximally mixed, and

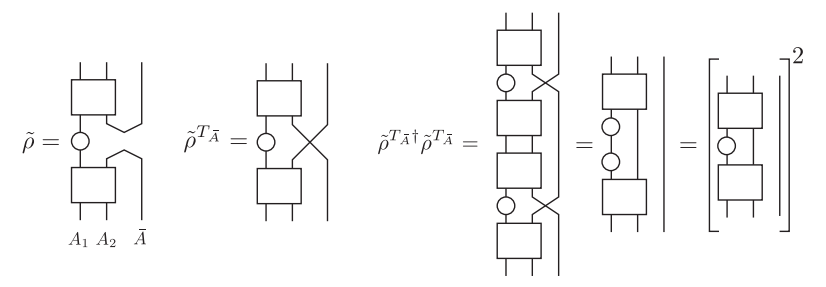

FIG. 3. Graphical representations of Eq. (13). Here, squares represent $U_{A}$ or $U_{A}^{\dagger}$, and circles represent $\rho_{A_{1}}$ we find no difference from the von Neumann entropy. However, when we move to AdS/CFT, the spectrum is not flat, and this term accounts for the tension of the cosmic brane. These codes are also not entirely satisfactory because there is no quantum correction to the logarithmic negativity.

Because $|\chi\rangle$ for the three-qutrit code is maximally entangled, we can apply (16). When bipartitioning the boundary, the bulk minimal geodesic cuts only a single leg (Fig. 4) of dimension 3, leading to a negativity of $\log (3)$.

\section{B. Subsystem QEC with complementary recovery}

Subsystem quantum error correction is a generalization to conventional quantum error correction. This generalization is crucial to our analysis because the holographic codes that we will employ belong to this family of errorcorrecting codes. There is a further generalization that is referred to as operator-algebra quantum error-correcting codes, though we leave this analysis to the Appendix A because it may distract from our main results. Again, we will make the Hilbert space $\mathcal{H}$ factorize into $\mathcal{H}_{A} \otimes \mathcal{H}_{\bar{A}}$, while the code subspace factorizes as $\mathcal{H}_{\text {code }}=\mathcal{H}_{a} \otimes \mathcal{H}_{\bar{a}}$. This code subspace is created such that the state can be recovered either on $A$ or $\bar{A}$. This construction allows the RT formula to be symmetric. The code space is spanned by

$$
|\tilde{i j}\rangle=U_{A}\left(|i\rangle_{A_{1}}\left|\chi_{j}\right\rangle_{A_{2}, \bar{A}}\right)=U_{\bar{A}}\left(|j\rangle_{\bar{A}_{1}}\left|\chi_{i}\right\rangle_{\bar{A}_{2}, A}\right) .
$$

We can simplify the code subspace to

$$
|\tilde{i j}\rangle=U_{A} U_{\bar{A}}\left(|i\rangle_{A_{1}}|j\rangle_{\bar{A}_{1}}|\chi\rangle_{A_{2} \bar{A}_{2}}\right)
$$

because

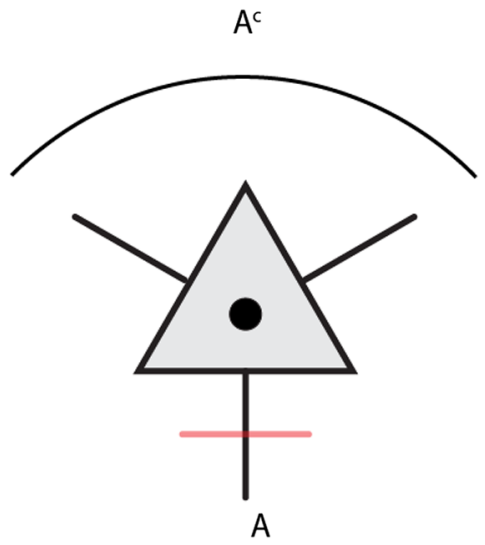

FIG. 4. The tensor network representation of the three-qutrit code. There is only one tensor in this network. It maps the single bulk logical qutrit (central black dot) to the three physical qutrits. The red line represents the minimal geodesic separating boundary region $A$ and its complement $A^{c}$. 


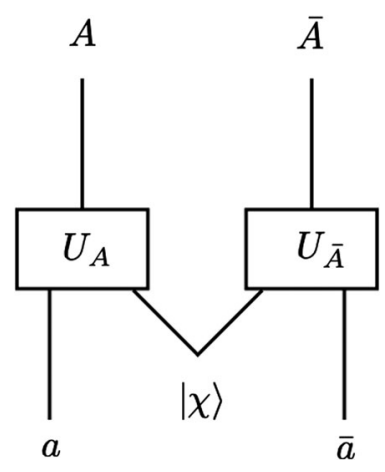

FIG. 5. In subsystem quantum error correction with complementary recovery, "bulk" degrees of freedom (d.o.f.) in the code subspace $\left(\mathcal{H}_{a} \otimes \mathcal{H}_{\bar{a}}\right)$ are encoded in the "boundary" Hilbert space $\left(\mathcal{H}_{A} \otimes \mathcal{H}_{\bar{A}}\right)$ using the auxiliary state $|\chi\rangle$ as an entanglement resource.

$$
\begin{aligned}
U_{\bar{A}}^{\dagger}\left|\chi_{j}\right\rangle_{A_{2}, \bar{A}} & =|j\rangle_{\bar{A}_{1}}|\chi\rangle_{A_{2} \bar{A}_{2}}, \\
U_{A}^{\dagger}\left|\chi_{i}\right\rangle_{\bar{A}_{2}, A} & =|i\rangle_{A_{1}}|\chi\rangle_{A_{2} \bar{A}_{2}} .
\end{aligned}
$$

Therefore, a density matrix can be encoded as

$$
\tilde{\rho}=U_{A} U_{\bar{A}}\left(\rho_{A_{1} \bar{A}_{1}} \otimes|\chi\rangle\left\langle\left.\chi\right|_{A_{2} \bar{A}_{2}}\right) U_{\bar{A}}^{\dagger} U_{A}^{\dagger} .\right.
$$

See Fig. 5 for a graphical representation. By defining $\chi_{A_{2}} \equiv$ $\operatorname{Tr}_{\bar{A}_{2}}|\chi\rangle\langle\chi|$ and $\chi_{\bar{A}_{2}} \equiv \operatorname{Tr}_{A_{2}}|\chi\rangle\langle\chi|$, we obtain

$$
\begin{aligned}
& \tilde{\rho}_{A}=U_{A}\left(\rho_{A_{1}} \otimes \chi_{A_{2}}\right) U_{A}^{\dagger}, \\
& \tilde{\rho}_{\bar{A}}=U_{\bar{A}}\left(\rho_{\bar{A}_{1}} \otimes \chi_{\bar{A}_{2}}\right) U_{\bar{A}}^{\dagger} .
\end{aligned}
$$

The associated area operators are

$$
\mathcal{L}_{A} \equiv S\left(\chi_{A_{2}}\right) I_{a}, \quad \mathcal{L}_{\bar{A}} \equiv S\left(\chi_{A_{2}}\right) I_{\bar{a}}
$$

so the new RT formulas are symmetric:

$$
S\left(\tilde{\rho}_{A}\right)=\left\langle\mathcal{L}_{A}\right\rangle+S\left(\tilde{\rho}_{a}\right), \quad S\left(\tilde{\rho}_{\bar{A}}\right)=\left\langle\mathcal{L}_{\bar{A}}\right\rangle+S\left(\tilde{\rho}_{\bar{a}}\right) .
$$

The calculation of negativity in subsystem quantum error correction with complementary recovery is quite similar to that of conventional QEC. We Schmidt decompose $|\chi\rangle_{A_{2} \bar{A}_{2}}$ as

$$
|\chi\rangle_{A_{2} \bar{A}_{2}}=\sum_{a} \sqrt{p_{a}}|a\rangle_{A_{2}}|a\rangle_{\bar{A}_{2}}
$$

so that the density matrix is

$\tilde{\rho}=U_{A} U_{\bar{A}}\left(\rho_{A_{1} \bar{A}_{1}} \otimes \sum_{a b} \sqrt{p_{a} p_{b}}|a\rangle\left\langle\left. b\right|_{A_{2}} \otimes \mid a\right\rangle\left\langle\left. b\right|_{\bar{A}_{2}}\right) U_{\bar{A}}^{\dagger} U_{A}^{\dagger}\right.$.

We now take the partial transpose with respect to $\bar{A}$ :

$$
\begin{aligned}
\tilde{\rho}^{T_{\bar{A}}}= & U_{A} U_{\bar{A}}^{T}\left(\rho _ { A _ { 1 } \overline { A } _ { 1 } } ^ { T _ { \overline { A } _ { 1 } } } \otimes \sum _ { a b } \sqrt { p _ { a } p _ { b } } | a \rangle \left\langle\left.b\right|_{A_{2}}\right.\right. \\
& \otimes|b\rangle\left\langle\left. a\right|_{\bar{A}_{2}}\right) U_{\bar{A}}^{\dagger T} U_{A}^{\dagger} .
\end{aligned}
$$

Taking the trace norm, we find

$$
\left|\tilde{\rho}^{T_{\bar{A}}}\right|_{1}=\left(\sum_{a} \sqrt{p_{a}}\right)^{2}\left|\rho_{A_{1} \bar{A}_{1}}^{T_{\bar{A}_{1}}}\right|_{1} .
$$

Therefore,

$$
\mathcal{E}(\tilde{\rho})=S_{1 / 2}\left(\chi_{A_{2}}\right)+\mathcal{E}\left(\rho_{A_{1}, \bar{A}_{1}}\right)=\langle\mathcal{L}\rangle+\mathcal{E}\left(\rho_{A_{1}, \bar{A}_{1}}\right)
$$

because $\chi_{A_{2}}$ is maximally mixed. Again, we have found the negativity to have a contribution from the area operator. However, this time, there is an additional quantum correction term equal to the negativity of the input state. We have thus found a quantum-corrected holographic logarithmic negativity formula. The quantum correction term is negligible when the bulk correction to the holographic von Neumann entropy (1) is negligible. We again note that the appearance of $S_{1 / 2}$ will imply nontrivial backreaction when we move to AdS/CFT.

\section{Entanglement negativity in holographic perfect tensor network codes}

So far, we have been working abstractly in the language of erasure-error-correcting codes. In order to obtain "geometric" insights of the entanglement structure of our quantum states, we now apply the results to the holographic perfect tensor network codes introduced in Ref. [10].

Holographic perfect tensor network codes are subsystem quantum-error-correcting codes made out of perfect tensors. They act as maps from the bulk Hilbert space of logical indices to the boundary Hilbert space of physical indices. The authors of Ref. [10] were able to analyze these codes from the perspective of a discrete RT formula by implementing the "greedy algorithm," which gives a corresponding "greedy geodesic." The greedy geodesic is initialized at a boundary subspace $A$. The greedy algorithm is implemented by removing tensors in the bulk one by one if more indices of that tensor lie outside of the greedy geodesic than inside. On a graph of negative curvature, this process will stop at some equilibrium position within the bulk, defining the greedy geodesic $\gamma_{A}$ for boundary subspace $A$. The graph version of the entanglement wedge is then the union of the tensors that are bounded by $A$ and $\gamma_{A}$.

In the following, we will analyze the logarithmic negativity of the holographic perfect tensor network codes. As the usefulness of negativity arises when working with mixed states, once again, we are mainly interested in the following two setups: (i) we start with a mixed state in the total (boundary) Hilbert space and then bipartition 
the boundary Hilbert space and discuss the entanglement negativity of the bipartition, and (ii) we start with a pure state but trace out a sub-Hilbert space to obtain a mixed state for the compliment. We then bipartition the remaining Hilbert space and discuss the entanglement negativity.

\section{Bipartite entanglement at finite temperature}

For the first setup, we put our boundary theory at finite temperature by introducing a black hole in the center of the bulk (see Fig. 6). Following Refs. [8,10], we implement the black hole by removing the central tensor. The new central legs are bulk indices that model the black hole entropy. The resulting tensor network is a subsystem quantum-errorcorrecting code. Therefore, we are able to apply the result from (28) to calculate the negativity of the bipartition. For the case of the entanglement entropy, the minimal cut homologous to $A$ "goes through" the black hole, and hence the entanglement entropy receives two types of contributions: the "quantum" part contributed from the part of the cut which does not "touch" the horizon and the "thermal" part coming from the horizon. For the case of the entanglement negativity, Eq. (28) suggests that we simply remove the thermal contribution, and consequently, it does not pick up the volume law contribution from the horizon. This tensor network picture of finite temperature holographic codes resembles the minimal entanglement wedge area of black holes (Fig. 1).

\section{Tripartite entanglement}

For the second setup, we investigate mixed states created by tracing out a subspace of an overall pure state (i.e., the bulk input state is pure). In doing so, we decompose the original error-correcting code into one that only has the physical d.o.f. in boundary subsystems $A$ and $B$. In order to arrive at this effective error-correcting code, we must trace out the d.o.f. of $C$ as seen in Fig. 7. This involves removing all of the tensors in the entanglement wedge of $C$ by

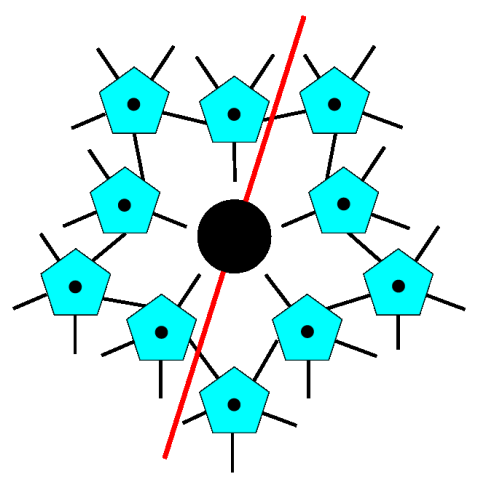

FIG. 6. A black hole in a holographic code is implemented by removing the central tensor of the network. The minimal geodesic (red) homologous to $A$ does not pick up any contributions from the black hole horizon and represents the entanglement wedge cross section.

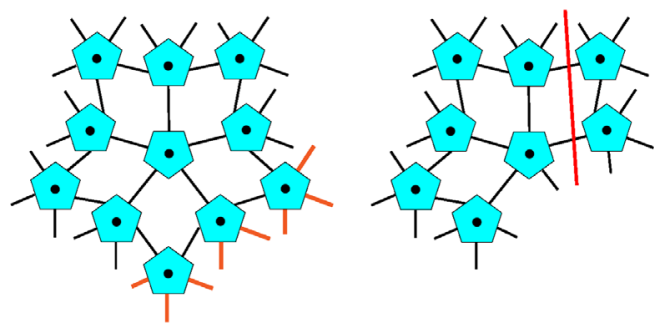

FIG. 7. The process of tracing out boundary subregion $C$ (orange indices on the left) to arrive at an effective errorcorrecting code (right) without disturbing $\rho_{A B}$. The red line on the right is the area term for the effective code, representing the entanglement wedge cross section.

repeatedly applying the Hermitian conjugates, $V^{\dagger}$, of the perfect tensors in the entanglement wedge of $C$. Once this process is completed, we are left with a new tensor network with a simplified geometry. The new tensor network is an isometry from the bulk logical indices to $A$ and $B$. The dangling bonds can be treated as an effective horizon analogous to the black hole horizon in the previous section. We are then able to repeat our argument from before and find the negativity to be equivalent to the entanglement wedge cross section in holographic codes with the bulk quantum correction [60].

\section{CONJECTURE FOR AdS/CFT}

The QEC code considerations above suggest that the logarithmic negativity is captured by the minimal entanglement wedge cross section. We now need to address the differences between tensor networks and AdS/CFT. For example, the spectrum of the entanglement Hamiltonian in holographic code models is completely flat (i.e., $|\chi\rangle$ is maximally entangled), while it is not in (holographic) CFTs. This implies that in the full-fledged AdS/CFT the area contribution in (28) should describe some backreacted geometry analogous to the area contribution for the holographic duals of Rényi entropies [61-63].

\section{A. Backreaction}

To address the issue of backreaction, we briefly overview Dong's proposal for the holographic dual of Rényi entropy. There, a close variant of the Rényi entropy is equal to the area of a cosmic brane with tension

$$
n^{2} \frac{\partial}{\partial n}\left(\frac{n-1}{n} S_{n}\right)=\frac{\text { Area }\left(\text { cosmic }_{\text {brane }}\right)}{4 G_{N}},
$$

where $S_{n}$ is the $n$th Rényi entropy and cosmic branes are gravitating objects living in the bulk. The tension of the cosmic brane depends on the replica index as

$$
T_{n}=\frac{n-1}{4 n G_{N}} .
$$


The cosmic brane is analogous to the RT surface except that it creates a conical deficit angle

$$
\Delta \phi=2 \pi \frac{n-1}{n} .
$$

In order to find the corresponding backreacted geometry, one must find the classical solution to the equations of motion for the action

$$
I=-\frac{1}{16 \pi G_{N}} \int d^{d+1} x \sqrt{G} R+I_{\text {matter }}+I_{\text {brane }}
$$

where

$$
I_{\text {brane }}=T_{n} \int d^{d-1} y \sqrt{g} .
$$

$G$ is the total bulk metric, while $g$ is the induced metric on the brane. Note that the brane becomes tensionless in the replica limit $(n \rightarrow 1)$, so the formula naturally reproduces the RT formula.

For negativity, we introduce backreaction in the bulk by defining a family of area functions in the ambient bulk of the entanglement wedge

$\left.\tilde{\mathcal{A}}_{n} \equiv n^{2} \frac{\partial}{\partial n}\left(\frac{n-1}{n} \mathcal{A}_{n}\right)=\frac{\text { Area }(\text { cosmic brane }}{n}\right)$,

where the bulk gravitational solution now has boundary conditions on the boundaries of the entanglement wedge. We then naturally claim

$$
\mathcal{E}=\lim _{n \rightarrow 1 / 2} \mathcal{A}_{n}+\mathcal{E}_{\text {bulk }}
$$

$\mathcal{A}_{n}$ is in general a very difficult problem to solve as one needs an analytic formula for $\tilde{\mathcal{A}}_{n}$. However, a special case of this is when the entangling surface is spherical, in which case we know the effect of the backreaction. In this special case, the negativity is proportional to the tensionless brane $(n \rightarrow 1)$ answer $[64,65]$

$$
\mathcal{E}=\mathcal{X}_{d}^{\text {hol }} \tilde{\mathcal{A}}_{1}+\mathcal{E}_{\text {bulk }}=\mathcal{X}_{d}^{\text {hol }} \frac{E_{W}}{4 G_{N}}+\mathcal{E}_{\text {bulk }},
$$

where

$$
\begin{aligned}
\mathcal{X}_{d}^{\mathrm{hol}} & =\frac{1}{2} x_{d}^{d-2}\left(1+x_{d}^{2}\right)-1, \\
x_{d} & =\frac{2}{d}\left(1+\sqrt{1-\frac{d}{2}+\frac{d^{2}}{4}}\right) .
\end{aligned}
$$

We will use this simplification throughout this paper. Observe that when $d=2, \mathcal{X}_{2}^{\text {hol }}=3 / 2$, and this relation is consistent with (and follows alternatively from) the fact that
$\mathcal{E}_{A}=S_{1 / 2}\left(\rho_{A}\right)$ and $S_{n}\left(\rho_{A}\right)=(c / 6)(1+1 / n) \ln \ell / \epsilon$, where $\ell$ is the radius (size) of the region (interval) $A$. The coefficient $\mathcal{X}_{d}^{\text {hol }}$ smoothly interpolates between the $\mathcal{X}_{2}^{\text {hol }}=$ $3 / 2$ that we will use for $(1+1)$ d CFTs and $\mathcal{X}_{\infty}^{\text {hol }}=$ $e-1-1.718$. Notably, for the $\mathcal{N}=4$ supersymmetric Yang-Mills, $\mathcal{X}_{4}^{\text {hol }} \sim 1.674$.

\section{B. Connection to entanglement of purification}

The minimal entanglement wedge cross section was studied in Refs. [37,39] as an interesting measure of entanglement in mixed states. References $[37,39]$ identified properties of this measure and matched these properties to a list of correlation measures in quantum information theory. They decided upon the entanglement of purification. The entanglement of purification is a famously difficult quantity to obtain. It is also dependent on both quantum and classical correlations, differing from the negativity which only measures quantum correlations. Even so, the proposal for holographic EoP and our proposal for holographic negativity do not contradict one another. Rather, we identify $\tilde{\mathcal{A}}_{1}$ in (34) with the conjectured holographic EoP and note that the negativity and EoP will be proportional only when the entangling surface is spherical and not for generic configurations.

It is also worth mentioning that there is yet another proposal for holographic negativity which has been shown to produce the correct behaviors for adjacent subsystems and bipartite thermal systems of $(1+1)$ d CFTs [46,49]. (There is also a higher-dimensional version of this proposal.) The proposal relates the entanglement negativity in holographic CFTs to a proper combination of the bulk minimal surface areas (geodesics). For example, for the case of two adjacent intervals at zero temperature, it was proposed that the entanglement negativity is given by

$$
\mathcal{E}=\frac{3}{16 G_{N}}\left(\mathcal{L}_{A_{1}}+\mathcal{L}_{A_{2}}-\mathcal{L}_{A_{1}, A_{2}}\right),
$$

where $\mathcal{L}_{A_{1}, A_{2}}$ is the area of the codimension-2 extremal surface homologous to the union of $A_{1}$ and $A_{2}$. As a corollary of this conjectured formula, the holographic negativity is related to the mutual information of the two intervals as

$$
\mathcal{E}=\frac{3}{4} I\left(A_{1}, A_{2}\right)
$$

Overall, there seems to be an intriguing connection between three quantum information theoretical quantities in holographic theories: the entanglement negativity, the entanglement of purification, and the mutual information.

Unfortunately, computing the entanglement of purification would be rather difficult in general. Using random tensor networks [15], we can compare the three quantities of interest: entanglement negativity, entanglement of 
purification, and mutual information. We look at a tripartition of the boundary. It was stated in Ref. [39] that any such tripartition can be decomposed into Bell and GHZ-like states, up to unentangled states,

$$
\begin{aligned}
& U_{A} U_{B} U_{C}|\Psi\rangle_{A B C} \\
& =\left(|\Phi\rangle_{A_{1} B_{1}}\right)^{c}\left(|\Phi\rangle_{B_{2} C_{1}}\right)^{a}\left(|\Phi\rangle_{A_{2} C_{2}}\right)^{b}\left(|\mathrm{GHZ}\rangle_{A_{3} B_{3} C_{3}}\right)^{g},
\end{aligned}
$$

with

$$
\begin{aligned}
|\Phi\rangle_{A B} & =\frac{1}{\sqrt{p}} \sum_{i=0}^{p-1}|i\rangle_{A}|i\rangle_{B}, \\
|\mathrm{GHZ}\rangle_{A B C} & =\frac{1}{\sqrt{p}} \sum_{i=0}^{p-1}|i\rangle_{A}|i\rangle_{B}|i\rangle_{C} .
\end{aligned}
$$

It is straightforward to then show that the negativity and entanglement of purification of $A B$ both equal $(c+g) \ln p$ and half the mutual information equals $\left(c+\frac{g}{2}\right) \ln p$. All three of these are coincident in the limit of large index dimension, $E_{P}(A, B)=\mathcal{E}=(1 / 2) I(A, B)$, which is the standard limit when dealing with random tensor networks.

\section{ENTANGLEMENT WEDGE AND NEGATIVITY IN AdS $\mathbf{A}_{3} / \mathrm{CFT}_{2}$}

In the following, we will make more detailed comparisons between the entanglement negativity and the minimal entanglement wedge cross section in the context of $\mathrm{AdS}_{3} /$ $\mathrm{CFT}_{2}$ : Specifically, the entanglement negativity here will be computed using the properties of holographic $\mathrm{CFT}_{2}$. When possible, we also compare these with (suitable linear combinations of) the mutual information. We recall that the minimal entanglement wedge cross section is proposed as a holographic dual of the entanglement of purification [37]; the proposed holographic formula relates the entanglement negativity to the mutual information [46]. We have seen that all these quantities in the specific setup of the random stabilizer code are equal.

Paralleling the discussion in tensor networks, we are interested in the following basic setups:

(1) The case of single interval.-In this case, we bipartition the total space into a single interval $A$ and its compliment $A^{c}$ and consider the entanglement negativity $\mathcal{E}^{A}$. The system can be in its ground state or in more generic pure or mixed states. However, our main focus will be cases of mixed states, in particular, the system at finite temperature, since for pure states the entanglement negativity is simply the Rényi entropy with Rényi index $1 / 2$.

(2) The case of two intervals.-In this case, we start from the ground state and tripartition the total system into intervals $A_{1}, A_{2}$, and $B$. We trace out $B$ and discuss the entanglement negativity of the reduced density matrix $\rho_{A_{1} A_{2}}$ for the two intervals $A_{1,2}$. The two intervals can be right next to each other (adjacent) or can be separated (disjoint) by the interval $B$.

(3) In addition, we will consider the entanglement negativity of the thermofield double state; here, we take the partial transpose in either one of the Hilbert spaces and discuss the entanglement negativity.

Let us warm up by considering a single interval at zero temperature. As previously mentioned, the entanglement negativity of the interval is equal to the Rényi entropy at Rényi index $1 / 2$. For $1+1 \mathrm{~d}$ CFTs, the Rényi entropies are simply determined by the central charge

$$
S_{n}=\frac{c}{12}\left(1+\frac{1}{n}\right) \ln \left(\frac{\ell}{\epsilon}\right),
$$

where $\ell$ is the length of the interval and $\epsilon$ is a UV cutoff, and hence in this case, the negativity is given by

$$
\mathcal{E}=S_{1 / 2}=\frac{c}{4} \ln \frac{\ell}{\epsilon} .
$$

Noting that the minimal entanglement wedge cross section in this case is equal to the length of the RT surface (equal to the von Neumann entanglement entropy), we confirm that

$$
\mathcal{E}=\frac{3}{2} E_{W}
$$

\section{A. Two intervals}

\section{Adjacent intervals}

We start with the entanglement negativity at zero temperature for two intervals $A_{1,2}$, which can be adjacent or disjoint. Our starting point is the expression of the moment $\operatorname{Tr}\left(\rho^{T_{2}}\right)^{n_{e}}$ as a correlation function of the twist operators $[16,17]$ :

$$
\begin{aligned}
\mathcal{E}= & \lim _{n_{e} \rightarrow 1} \ln \operatorname{Tr}\left(\rho^{T_{2}}\right)^{n_{e}} \\
= & \lim _{n_{e} \rightarrow 1} \ln \left\langle\sigma_{n_{e}}\left(w_{1}, \bar{w}_{1}\right) \bar{\sigma}_{n_{e}}\left(w_{2}, \bar{w}_{2}\right)\right. \\
& \left.\times \bar{\sigma}_{n_{e}}\left(w_{3}, \bar{w}_{3}\right) \sigma_{n_{e}}\left(w_{4}, \bar{w}_{4}\right)\right\rangle_{\mathbb{C}} .
\end{aligned}
$$

Here, the conformal dimension of the twist operator $\sigma_{n}$ is

$$
h_{n}=\frac{c}{24}\left(n-\frac{1}{n}\right) \text {. }
$$

The complex Euclidean coordinates $w=i \tau+x$ are set to be $w_{1}=Y_{1}, w_{2}=Y_{2}, w_{3}=X_{1}, w_{4}=X_{2}$, with

$$
X_{1}-X_{2}=\ell_{1}, \quad Y_{1}-Y_{2}=\ell_{2}, \quad Y_{2}-X_{1}=d,
$$


where $\ell_{1,2}$ is the length of the interval $A_{1,2}$ and $d$ is the distance between the intervals.

In the limit of the adjacent intervals, $d \rightarrow 0$, the negativity is given by the three-point function,

$\mathcal{E}=\lim _{n_{e} \rightarrow 1} \ln \left\langle\sigma_{n_{e}}\left(w_{1}, \bar{w}_{1}\right) \bar{\sigma}_{n_{e}}^{2}\left(w_{2}, \bar{w}_{2}\right) \sigma_{n_{e}}\left(w_{4}, \bar{w}_{4}\right)\right\rangle_{\mathbb{C}}$,

and hence completely determined by conformal symmetry. Using the dimension of the twist operator, one then obtains

$$
\mathcal{E}=\frac{c}{4} \ln \left[\frac{\ell_{1} \ell_{2}}{\ell_{1}+\ell_{2}}\right]+\text { const. }
$$

Let us compare the negativity (49) with the minimal cross section of the corresponding entanglement wedge, which is given, according to Ref. [37], by

$$
E_{W}= \begin{cases}\frac{c}{6} \ln \frac{1+\sqrt{x}}{1-\sqrt{x}}, & \frac{1}{2} \leq x \leq 1 \\ 0, & 0 \leq x \leq \frac{1}{2}\end{cases}
$$

where $x$ is the cross-ratio,

$$
x:=\frac{w_{12} w_{34}}{w_{13} w_{24}}=\frac{\ell_{1} \ell_{2}}{\left(\ell_{1}+d\right)\left(\ell_{2}+d\right)} .
$$

In the limit of adjacent intervals $d \rightarrow 0$,

$$
E_{W} \rightarrow \frac{c}{6} \ln (4 z)=\frac{c}{6} \ln \left(\frac{4}{\epsilon} \frac{\ell_{1} \ell_{2}}{\epsilon\left(\ell_{1}+\ell_{2}\right)}\right) .
$$

Thus, if the constant in (49) is properly chosen, $\mathcal{E}=(3 / 2) E_{W}$.

Let us also consider the following, properly normalized, mutual information for the two intervals: $(3 / 4) I\left(A_{1}, A_{2}\right)$. This quantity was claimed to be equal to the entanglement negativity in AdS/CFT [46]. This claim follows from the proposed holographic formula for the entanglement negativity for the mixed state of the adjacent intervals

$$
\mathcal{E}=\frac{3}{4} \cdot \frac{1}{4 G_{N}}\left[\mathcal{L}_{12}+\mathcal{L}_{23}-\mathcal{L}_{13}\right]
$$

where $\mathcal{L}_{12}$, etc., are the bulk geodesic lengths. It is straightforward to check that $(3 / 4) I\left(A_{1}, A_{2}\right)$ is also given by $(c / 4) \ln \left[\ell_{1} \ell_{2} /\left(\ell_{1}+\ell_{2}\right)\right]+$ const. Summarizing, for adjacent intervals, all three quantities are equal,

$$
\mathcal{E}=\frac{3}{2} E_{W}=\frac{3}{4} I\left(A_{1}, A_{2}\right) .
$$

We now generalize to a thermal state. We take adjacent intervals of equal length $\ell$. For finite temperature, the negativity of adjacent intervals is computed by the following three-point function of twist fields on the cylinder:

$$
\mathcal{E}=\lim _{n_{e} \rightarrow 1} \ln \left(\left\langle\sigma_{n_{e}}\left(z_{1}\right) \bar{\sigma}_{n_{e}}^{2}\left(z_{2}\right) \sigma_{n_{e}}\left(z_{3}\right)\right\rangle_{\beta}\right)
$$

Unlike the case for thermal bipartite negativity, there are no ambiguities regarding transforming from the complex plane to the cylinder. This is due to the adjacent intervals being finite [66]. We use the following map from the complex plane to the cylinder,

$$
\begin{aligned}
w(z) & =e^{2 \pi z / \beta} \\
\mathcal{E} & =\left(\frac{2 \pi}{\beta}\right)^{-c / 4}\left\langle\sigma\left(e^{-2 \pi \ell / \beta}\right) \bar{\sigma}^{2}(1) \sigma\left(e^{2 \pi \ell / \beta}\right)\right\rangle_{\mathbb{C}},
\end{aligned}
$$

where we have taken the replica limit. We then compute the three-point function to arrive at a negativity of

$$
\mathcal{E}=\frac{c}{4} \ln \left[\frac{\beta}{2 \pi \epsilon} \tanh \left(\frac{\pi \ell}{\beta}\right)\right],
$$

where we have introduced the regulator $\epsilon$.

We can now do the corresponding calculation holographically. We use the planar BTZ geometry

$$
d s^{2}=-\frac{\left(r^{2}-r_{H}^{2}\right)}{R^{2}} d t^{2}+\frac{d r^{2}}{r^{2}-r_{H}^{2}}+\frac{r^{2}}{R^{2}} d x^{2} .
$$

Due to the symmetry of the setup, the minimal cross section is purely radial,

$$
\Sigma=\int_{r_{*}}^{r_{\infty}} \frac{d r}{\sqrt{r^{2}-r_{H}^{2}}},
$$

where $r_{*}$ is the location of the turning point which is related to the interval length by [5]

$$
r_{*}=r_{H} \operatorname{coth}\left(\ell r_{H}\right)
$$

Using (28), we arrive at

$$
\mathcal{E}=\frac{3}{8 G_{N}} \Sigma=\frac{c}{4} \ln \left[\frac{\beta}{2 \pi \epsilon} \tanh \left(\frac{\pi \ell}{\beta}\right)\right],
$$

which exactly matches the CFT result. We note that the same answer has been found using (53) [46].

\section{Disjoint intervals}

While the negativity for adjacent intervals is given in terms of the three-point function and is hence universal, the negativity for disjoint intervals depends on the full operator content of the theory. Let us examine in the case of the holographic CFT in the large- $c$ limit, using the result from Ref. [67].

Starting from (45), using a conformal map that sends $w_{1} \rightarrow \infty, w_{2} \rightarrow 1, w_{3} \rightarrow x$, and $w_{4} \rightarrow 0$, the negativity is written as 


$$
\begin{aligned}
\mathcal{E}= & \lim _{n_{e} \rightarrow 1} \ln \left(\left|w_{24}\right|^{-4 h_{n_{e}}}\left|w_{13}\right|^{-4 h_{n_{e}}}\right) \\
& +\lim _{n_{e} \rightarrow 1} \ln \left[\left\langle\sigma_{n_{e}}(\infty) \bar{\sigma}_{n_{e}}(1) \bar{\sigma}_{n_{e}}(x, \bar{x}) \sigma_{n_{e}}(0)\right\rangle\right] .
\end{aligned}
$$

The first term does not contribute in the replica limit since $h_{n_{e}} \rightarrow 0$. Hence, the sole contribution [the second term in (62)] depends only on $x$, and the negativity for two disjoint intervals (at zero temperature and for infinite systems) is a scale invariant quantity.

Monodromy method.-We now try to find behaviors of the universal function $\left\langle\sigma_{n_{e}}(\infty) \bar{\sigma}_{n_{e}}(1) \bar{\sigma}_{n_{e}}(x, \bar{x}) \sigma_{n_{e}}(0)\right\rangle$ in the large- $c$ limit. It can be expanded in terms of the conformal blocks as

$$
\begin{aligned}
& \left\langle\sigma_{n_{e}}(\infty) \bar{\sigma}_{n_{e}}(1) \bar{\sigma}_{n_{e}}(x, \bar{x}) \sigma_{n_{e}}(0)\right\rangle \\
& \quad=\sum_{p} a_{p} \mathcal{F}\left(c, h_{p}, h_{i}, x\right) \overline{\mathcal{F}}\left(c, \bar{h}_{p}, \bar{h}_{i}, \bar{x}\right),
\end{aligned}
$$

where $p$ labels operators in intermediate operator product expansion channels with conformal dimension $h_{p} ; h_{i}$ collectively represents the conformal dimensions of four "external" operators, i.e., $h_{n_{e}} ; a_{p}$ is a constant depending on the operator product expansion coefficients. In the decomposition of the conformal block, we assume there is a single dominant channel $p$ and disregard other contributions [68,69]:

$$
\begin{aligned}
& \left\langle\sigma_{n_{e}}(\infty) \bar{\sigma}_{n_{e}}(1) \bar{\sigma}_{n_{e}}(x, \bar{x}) \sigma_{n_{e}}(0)\right\rangle \\
& \quad \sim \mathcal{F}\left(c, h_{p}, h_{i}, x\right) \overline{\mathcal{F}}\left(c, \bar{h}_{p}, \bar{h}_{i}, \bar{x}\right) .
\end{aligned}
$$

For holographic CFTs, the conformal block exponentiates as [70]

$$
\mathcal{F}\left(c, h_{p}, h_{i}, x\right) \sim \exp \left[-\frac{c}{6} f\left(\frac{h_{p}}{c}, \frac{h_{i}}{c}, x\right)\right] .
$$

Hence, assuming $f\left(h_{p} / c, h_{i} / c, x\right)=f\left(\bar{h}_{p} / c, \bar{h}_{i} / c, \bar{x}\right)$,

$$
\ln \left\langle\sigma_{n_{e}}(\infty) \bar{\sigma}_{n_{e}}(1) \bar{\sigma}_{n_{e}}(x, \bar{x}) \sigma_{n_{e}}(0)\right\rangle \sim-\frac{c}{3} f\left(\frac{h_{p}}{c}, \frac{h_{i}}{c}, x\right) .
$$

In Ref. [67], the dominant channel when $x \rightarrow 1$ (the limit of adjacent intervals $d / \ell_{1,2} \rightarrow 0$ ) is identified as the double twist operator $\sigma_{n_{e}}^{2}$ with conformal dimension $h_{p}=h_{n_{e}}^{(2)}=$ $(c / 12)\left(n_{e} / 2-2 / n_{e}\right)$. On the other hand, when $x \rightarrow 0$ (the limit where the distance between of two intervals is large $\left.d / \ell_{1,2} \rightarrow \infty\right)$, the dominant channel is vacuum. The analysis in the latter case $(x \rightarrow 0)$ is similar (identical) to the case of the entanglement entropy of two disjoint intervals; it is exponentially small. (For small $x$, the computation of the four-point function is identical to the one performed for entanglement entropy, and there is a factor of $(n-1)$ which vanishes in the $n \rightarrow 1$ limit.) In the following, we will mainly focus on the case of $x \rightarrow 1$.

The function $f$ can be found by using the monodromy method, and this program was carried out in Ref. [67]. The same kind of approximation was used to compute the mutual information for disjoint intervals in holographic CFT in Ref. [68] to reproduce the result from the RT formula. There, as the distance between the two intervals increases/decreases, there is a "phase transition," and the mutual information has a "singularity" as a function of the distance between the intervals [71]. We expect there is a similar phase transition in the entanglement negativity [67].

In the monodromy method, the large- $c$ conformal block $f$ is given in terms of the accessory parameter $c_{2}$ as $\partial f / \partial x=c_{2}(x)$. In Ref. [67], two solutions were found numerically in the monodromy problem and are approximately given by

$$
\begin{aligned}
& y(1-y) c_{2}^{ \pm}(1-y)=-\frac{3}{4}+\frac{3}{4}\left(\frac{1}{2} \pm \frac{1}{4}\right) y+\cdots, \\
& c_{2}^{-}(x) \sim \frac{3(x+3)}{16 x(x-1)}, \quad c_{2}^{+}(x) \sim \frac{3(3 x+1)}{16 x(x-1)},
\end{aligned}
$$

where $y=1-x$. By integrating $c_{2}$, these accessory parameters are translated to conformal blocks in the vicinity of $x=1$,

$$
\begin{aligned}
& f^{-}(x)=\frac{3}{16} \ln \left[\frac{(1-x)^{4}}{x^{3}}\right]+\text { const. } \\
& f^{+}(x)=\frac{3}{16} \ln \left[\frac{(1-x)^{4}}{x}\right]+\text { const. }
\end{aligned}
$$

If the dominant solution $\left[f^{-}(x)\right.$ in this case $]$ is chosen, the entanglement negativity is given by

$$
\mathcal{E}^{-} \sim-\frac{c}{3} \cdot \frac{3}{16} \ln \left[\frac{(1-x)^{4}}{x^{3}}\right]+\text { const. }
$$

As in the case of adjacent intervals, the constant has to be chosen properly, which cannot be determined from the monodromy method. We set

$$
\text { const }=\frac{c}{4} \ln (4) .
$$

[See Eqs. (49) and (52) in Sec. IV A 1.]

The entanglement negativity, computed by using the solutions $f^{ \pm}$, is plotted in Fig. 8, together with the minimal entanglement wedge cross section. Note that the above solutions are valid for $x \sim 1$. On the other hand, for sufficiently small $x$, there is a phase transition to the other branch, where negativity is simply zero. While the entanglement negativity $\mathcal{E}^{ \pm}$and the minimal entanglement 


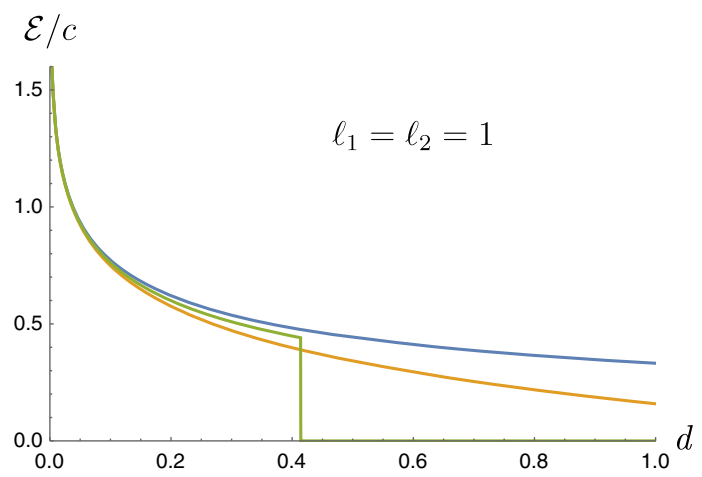

FIG. 8. (Blue and yellow) The logarithmic negativity for disjoint intervals at zero temperature for holographic CFT computed from the large- $c$ conformal blocks $f^{ \pm}(x)$ as a function of the distance $d$ between the two intervals. (Green) The minimal entanglement wedge cross section, $(3 / 2) E_{W}$, plotted in the unit of $c$.

wedge cross section $(3 / 2) E_{W}$ disagree, it is interesting to note that the minimal entanglement wedge cross section is right in between the two solutions.

To have a closer comparison with the minimal entanglement wedge cross section $(3 / 2) E_{W}$ (52), we define an analogue of conformal block $\mathcal{F}^{W}(x)$ by

$$
\frac{3}{2} E_{W}(x)=: \ln \left[a_{p} \mathcal{F}^{W}(x) \overline{\mathcal{F}}^{W}(x)\right] .
$$

Choosing $a_{p}=4^{c / 4}$ [i.e., $\ln a_{p}=(c / 4) \ln (4)$; see (70)],

$$
\mathcal{F}^{W}(x)=\left[\frac{1}{4} \frac{1+\sqrt{x}}{1-\sqrt{x}}\right]^{\frac{c}{8}} .
$$

$\mathcal{F}^{W}(x)$ can be expanded in small $y=1-x$ as

$$
\begin{aligned}
\mathcal{F}^{W}(x)= & y^{-\frac{c}{8}}\left[1-\frac{c y}{16}+\frac{c(c-12)}{512} y^{2}\right. \\
& \left.-\frac{c\left(c^{2}-36 c+320\right)}{24576} y^{3}+\cdots\right] .
\end{aligned}
$$

Further introducing the corresponding accessory parameter by

$$
c_{2}^{W}(x):=-\frac{3}{4} \frac{6}{c} \frac{d E_{W}}{d x}=-\frac{3}{4} \frac{1}{(1-x) \sqrt{x}},
$$

we see that $c_{2}^{-}$and $c_{2}^{W}$ disagree at linear order in $y$ :

$$
y(1-y) c_{2}^{W}(1-y)=-\frac{3}{4}+\frac{3}{8} y+\frac{3}{32} y^{2}+\cdots .
$$

Series expansion.-Reference [67] also looked at the expansion of the conformal block in terms of the cross-ratio,

$$
\begin{aligned}
\mathcal{F}\left(h_{p}, y\right) & =y^{h_{p}}\left[1+\frac{h_{p}}{2} y+\frac{h_{p}\left(h_{p}+1\right)^{2}}{4\left(2 h_{p}+1\right)} y^{2}\right. \\
& \left.+\frac{h_{p}^{2}\left(1-h_{p}\right)^{2}}{2\left(2 h_{p}+1\right)\left[c\left(2 h_{p}+1\right)+2 h_{p}\left(8 h_{p}-5\right)\right]} y^{2}+\cdots\right],
\end{aligned}
$$

where once again $y=1-x$. Setting $h_{p}=-c / 8$, we obtain

$$
\mathcal{F}\left(h_{p}, y\right)=y^{-\frac{c}{8}}\left[1-\frac{c y}{16}+\frac{(c-16) c y^{2}}{576}+\cdots\right] .
$$

This is supposed to be valid for any $c$, but as we will see, there is a complication. It seems that the $h_{p} \rightarrow-c / 8$ limit and the large- $c$ limit do not commute.

One reason is that, for generic values of $h_{p}$, the third term is of order $(c y)^{2}$ and the forth term is of order $c y^{2}$, while when $h_{p}=-c / 8$, they are both of the same order. On the other hand, for $h_{p} \sim a c$ with $c$ large and a generic value of $a \neq-1 / 8$, we keep leading order terms $(c y)^{n}$. For example, in the above expression (76), the third term is of order $(c y)^{2}$, while the last term is subleading and of order $c y^{2}$. Collecting the $(c y)^{n}$ terms,

$\mathcal{F}\left(h_{p}, y\right)=y^{h_{p}}\left[1+\frac{h_{p}}{2} y+\frac{h_{p}^{2}}{8} y^{2}+\frac{h_{p}^{3}}{48} y^{3}+\cdots\right]$.

On the other hand, from the entanglement wedge cross section, keeping leading order terms,

$$
\mathcal{F}^{W}(x)=y^{-\frac{c}{8}}\left[1-\frac{c y}{16}+\frac{c^{2} y^{2}}{512}-\frac{c^{3} y^{3}}{24576}+\cdots\right] .
$$

Substituting $a=-1 / 8$ in (78), Eq. (78) matches precisely with (79). Note also that (78) can be exponentiated as $\mathcal{F}\left(h_{p}, y\right)=\exp [-(c / 6) f(x)]$ with

$$
f(x)=-\frac{6}{c}\left[c\left(a \ln y+\frac{a}{2} y+\cdots\right)\right] .
$$

The corresponding accessory parameter is given by $c_{2}(x)=\partial f / \partial x=3 a+6 a /(1-x)$. Expanded in $y$ and substituting $a=-1 / 8$ naively,

$$
y(1-y) c_{2}(1-y)=-\frac{3}{4}+\frac{3}{8} y .
$$

This expansion matches with the expansion of the entanglement wedge cross section $c_{2}^{W}$ (75). This is consistent with the result from the monodromy method. However, of course, $a=-1 / 8$ is precisely the point where various complications arise, as seen from (76): some of the expansion coefficients in (76) diverge. 


\section{B. Single interval at finite temperature}

Let us now discuss the case of a single interval at finite temperature. In this case, the negativity can be expressed as [66]

$\mathcal{E}=\lim _{L \rightarrow \infty} \lim _{n_{e} \rightarrow 1} \ln \left[\left\langle\sigma_{n_{e}}(-L) \bar{\sigma}_{n_{e}}^{2}(-\ell) \sigma_{n_{e}}^{2}(0) \bar{\sigma}_{n_{e}}(L)\right\rangle_{\beta}\right]$,

where the conformal dimensions of $\sigma_{n_{e}}$ and $\sigma_{n_{e}}^{2}$ are given by

$$
\begin{aligned}
h_{n_{e}} & =\frac{c}{24}\left(n_{e}-\frac{1}{n_{e}}\right), \\
h_{n_{e}}^{(2)} & =2 h_{\frac{n_{e}}{2}}=\frac{c}{12}\left(\frac{n_{e}}{2}-\frac{2}{n_{e}}\right) .
\end{aligned}
$$

Here, the order of the limits is important; the replica limit has to be taken before the $L \rightarrow \infty$ limit. Below, we use the twist operator formula to compute the entanglement negativity. Noting $h_{n} \rightarrow 0$ and $h_{n}^{(2)} \rightarrow-c / 8$ in the replica limit, the negativity is given by

$\mathcal{E}=\frac{c}{2} \ln \left[\frac{\beta}{2 \pi} e^{\frac{\pi \ell}{\beta}}\right]+\lim _{L \rightarrow \infty} \lim _{n_{e} \rightarrow 1} \ln \left\langle\sigma_{n}(\infty) \bar{\sigma}_{n}^{2}(1) \sigma_{n}^{2}(x, \bar{x}) \bar{\sigma}_{n}(0)\right\rangle$.

Here, the cross-ratio in the $L \rightarrow \infty$ limit is

$$
x=\frac{\left(1-e^{\frac{2 \pi L}{\beta}}\right)\left(e^{-\frac{2 \pi L}{\beta}}-e^{-\frac{2 \pi \ell}{\beta}}\right)}{\left(e^{-\frac{2 \pi L}{\beta}}-1\right)\left(e^{-\frac{2 \pi \ell}{\beta}}-e^{\frac{2 \pi L}{\beta}}\right)} \rightarrow e^{-2 \pi \ell / \beta} .
$$

As for the conformal block part, one can derive its semiclassical approximation by using the monodromy method

$$
\ln \left\langle\sigma_{n}(\infty) \bar{\sigma}_{n}^{2}(1) \sigma_{n}^{2}(x, \bar{x}) \bar{\sigma}_{n}(0)\right\rangle=-\frac{c}{3} f(x),
$$

where $f(x)$ can be computed for $x \sim 0$ (s channel) and $x \sim 1$ ( $t$ channel) separately by using the monodromy method. See Ref. [72] for previous calculations.

\section{1. t channel}

In this channel, the identity block is dominant. The monodromy calculation is straightforward and gives

$$
f(x)=\frac{12 h_{n_{e}}^{(2)}}{c} \ln (1-x), \quad x \rightarrow 1 .
$$

This is a situation very similar to the entanglement entropy; the vacuum (identity) block is completely determined by the primary operator with no additional contributions from descendant operators. Recalling $x \rightarrow e^{-2 \pi \ell / \beta}$ in $L \rightarrow \infty$,

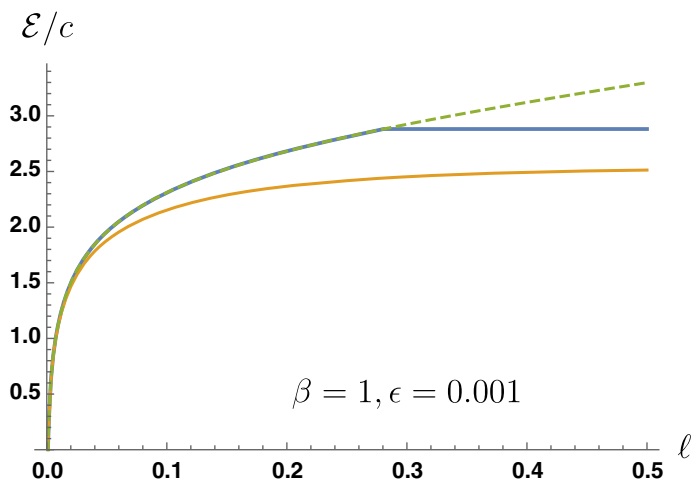

FIG. 9. The logarithmic negativity for an interval of length $\ell$ at finite temperature $\beta$ for holographic CFT. (Yellow) The generic CFT result (90) with $g=0$. (Green) The negativity (88) computed from the $t$-channel solution (87) for $x \sim 1$. (Blue) The minimal entanglement wedge cross section.

$$
\mathcal{E}=\frac{c}{2} \ln \frac{\beta}{2 \pi}+\frac{c}{2} \ln \left(2 \sinh \frac{\pi \ell}{\beta}\right), \quad x \rightarrow 1 .
$$

Note that the proper cutoff factor is missing in these expressions. We simply replace $\beta / 2 \pi \rightarrow \beta /(2 \pi \epsilon)$.

The above result can be compared with the minimal entanglement wedge cross section [37],

$$
\frac{3}{2} E_{W}=\frac{c}{2} \min \left[\ln \left(\frac{\beta}{\pi \epsilon} \sinh \frac{\pi \ell}{\beta}\right), \ln \left(\frac{\beta}{\pi \epsilon}\right)\right],
$$

and the generic CFT result [66]

$$
\mathcal{E}=\frac{c}{2} \ln \left(\frac{\beta}{\pi a_{0}} \sinh \frac{\pi \ell}{\beta}\right)-\frac{\pi c \ell}{2 \beta}+g\left(e^{-2 \pi \ell / \beta}\right)
$$

with $g=0$. With the choice $g=0$, this also is the expected result from the holographic negativity proposal $[46,49]$ and related to the mutual information. Near $x \sim 1$, all of these three quantities (the entanglement negativity, the minimal entanglement wedge cross section, and the mutual information) agree. See Fig. 9.

\section{2. s channel}

In this channel, the dominant operator is the twist operator $\sigma_{n}$ with dimension $h_{n}$. The semiclassical conformal block can be obtained by solving the monodromy problem around $(x, 0)$ with the trivial monodromy $\operatorname{Tr} M_{(x, 0)}=2$ in the replica limit.

Numerical solutions of the monodromy problem are shown in Fig. 10. The situation here is similar to the negativity for disjoint intervals for $x \sim 1$. [See Sec. IV A 2, around (67).] There are two solutions $c_{2}^{ \pm}$centered around

$$
c_{2}^{W}(x)=-\frac{3}{4} \frac{1}{x} .
$$




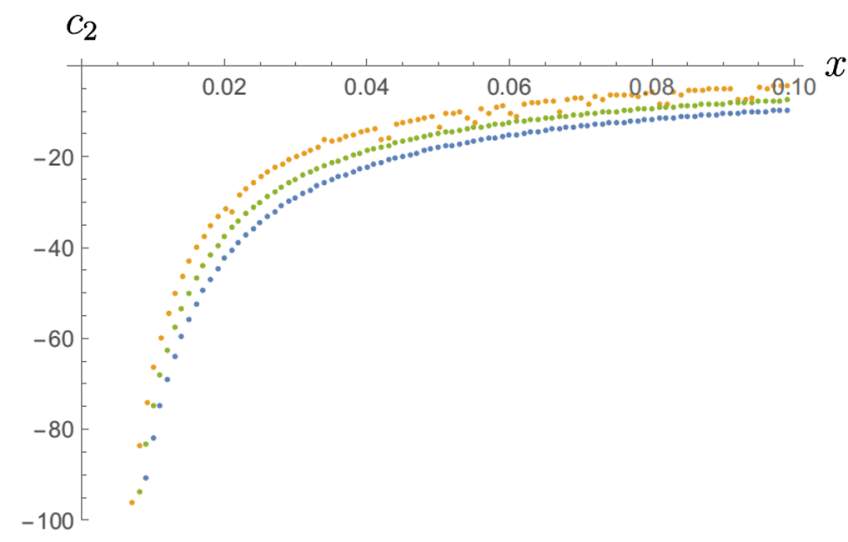

FIG. 10. The accessory parameter $c_{2}$ as a function of the crossratio $x$. There are two solutions (blue and yellow) centered around $c_{2}^{W}(x)=-3 /(4 x)$ (green).

The accessory parameter $c_{2}^{W}(x)$ is consistent with the minimal entanglement wedge cross section (89); the corresponding conformal block, up to a unknown constant, is given by

$$
\begin{aligned}
& f^{W}(x)=\frac{6 h_{n_{e}}^{(2)}}{c} \ln (x)=\frac{-3}{4} \ln x, \quad x \rightarrow 0, \\
& \mathcal{F}^{W}(x)=x^{\frac{c}{8}}
\end{aligned}
$$

If we use the conformal block $\mathcal{F}^{W}$ and $f^{W}(x)$, the negativity is constant as a function of $\ell / \beta$ for $x \sim 0$ :

$$
\mathcal{E}=\frac{c}{2} \ln \frac{\beta}{2 \pi}, \quad x \rightarrow 0
$$

Note that the proper cutoff factor is missing in this expression. We simply replace $\beta / 2 \pi \rightarrow \beta /(2 \pi \epsilon C)$, where $\epsilon$ is the cutoff and $C$ is an unknown constant. The negativity (93) can be matched with the minimal entanglement wedge cross section (89) by choosing $C$ properly $(C=2)$. On the other hand, with the solution $\mathcal{F}^{+}$or $\mathcal{F}^{-}$, the negativity is not constant for $x \sim 0$.

It is also worthwhile to have a look at the series expansion (76): with $h_{n}^{(2)}=a c, h_{n}=\delta c$, the series expansion gives

$$
\mathcal{F}(x)=x^{-a c}\left[1+\frac{a^{2} c x}{2 \delta}+\cdots\right]
$$

In the replica limit $a \rightarrow-c / 8$ and $\delta=0$, each term in the expansion diverges, except for the leading term. Keeping this term alone and discarding (heuristically) all divergent terms reproduce $\mathcal{F}^{W}$. Once again, this is a situation very similar to the case of the two disjoint intervals.

\section{Thermofield double state}

In this section, we consider the thermofield double state in CFT. It is a purification of the mixed thermal state at inverse temperature $\beta$ and given by

$$
|\mathrm{TFD}\rangle=\frac{1}{\sqrt{Z(\beta)}} \sum_{i} e^{-\beta E_{i} / 2}|i\rangle_{1}|i\rangle_{2},
$$

where we have introduced the two copies of the original CFT Hilbert space, $\mathcal{H}_{\text {tot }}=\mathcal{H}_{1} \otimes \mathcal{H}_{2}$, and $|i\rangle_{1,2}$ is the $i$ th energy eigenstate with energy $E_{i} ; Z(\beta)$ is the partition function. When tracing out either copy of the CFT, the resulting reduced density matrix is thermal. The thermofield double state is conjectured to be dual to the $\mathrm{AdS}$ eternal black hole [73].

We follow Ref. [65] to obtain the negativity between the copies. From the density matrix

$$
\rho_{\mathrm{TFD}}=\frac{1}{Z(\beta)} \sum_{i, j} e^{-\beta\left(E_{i}+E_{j}\right) / 2}|i\rangle_{1}\left\langle\left. j\right|_{1} \otimes \mid i\right\rangle_{2}\left\langle\left. j\right|_{2},\right.
$$

it is straightforward to compute

$$
\left|\rho_{\mathrm{TFD}}^{T_{1}}\right|_{1}=\frac{Z(\beta / 2)^{2}}{Z(\beta)} .
$$

By taking the logarithm, the entanglement negativity is given in terms of the free energy $F(\beta)=-(1 / \beta) \ln (Z(\beta))$ as

$$
\mathcal{E}\left(\rho_{\mathrm{TFD}}\right)=\beta[F(\beta)-F(\beta / 2)] .
$$

In the holographic pentagon code, we can create the AdS eternal black hole by connecting two codes with black holes at their center by linking the black hole microstate legs (Fig. 6). Using (28) and following our discussion in Sec. II C, we see that the negativity is given by the area of the horizon $A_{\mathrm{BH}}$ as

$$
\mathcal{E}\left(\rho_{\mathrm{TFD}}\right) \propto A_{\mathrm{BH}}
$$

leading us to an interesting relation between the black hole area/entropy and the temperature/free energy of the dual CFT,

$$
\beta[F(\beta)-F(\beta / 2)]_{\mathrm{CFT}} \propto A_{\mathrm{BH}} .
$$

When moving beyond the tensor network description to the full AdS/CFT, we analogously find the minimal cross sectional area of the entanglement wedge to be the area of the black hole horizon. In $\mathrm{AdS}_{3} / \mathrm{CFT}_{2}$, we adopt the same normalization constant (equal to $3 / 2$ ) as before relating the negativity and the minimal entanglement wedge cross section. This leads to 


$$
\begin{gathered}
\beta[F(\beta)-F(\beta / 2)]=\frac{3}{2} \frac{A_{\mathrm{BH}}}{4 G_{N}} \\
\text { or } \quad F(\beta)=F(\beta / 2)+\frac{3 A_{\mathrm{BH}}}{8 \beta G_{N}} .
\end{gathered}
$$

We implement this recursively to obtain

$$
F(\beta)=\frac{3}{8 G_{N}} \sum_{i=0}^{\infty} \frac{A_{\mathrm{BH}}\left(\beta / 2^{i}\right)}{\beta / 2^{i}}
$$

We work with the boundary of the Euclidean BTZ black hole, which is of length $L=2 \pi l_{\mathrm{AdS}}$ (where $l_{\mathrm{AdS}}$ is the radius of $\mathrm{AdS}$ ). Using $r_{\mathrm{BH}}=2 \pi / \beta$, we arrive at the formally divergent sum

$$
F(\beta)=\frac{3 \pi^{2} l_{\mathrm{AdS}}}{2 G_{N} \beta^{2}} \sum_{i=0}^{\infty} 4^{i} .
$$

We can obtain a value for this by analytically continuing the geometric series. This gives us a value of $-1 / 3$. We use the Brown-Henneaux formula to arrive at

$$
F(\beta)=\mathcal{O}\left(\frac{L}{\epsilon}\right)-\frac{\pi c L}{6 \beta^{2}}+\mathcal{O}(\epsilon)+\cdots,
$$

where $L$ is the size of the CFT system and $\epsilon$ is the cutoff. The finite, universal part of the free energy precisely matches that for a thermal CFT.

\section{DISCUSSION}

We have discussed negativity in quantum errorcorrecting codes and tensor network models of holography. We have shown that the entanglement negativity in these models is captured by the minimal cross sectional area of the entanglement wedge. We have also conjectured a generalization to AdS/CFT using the backreacted geometry of cosmic branes and have checked our proposal for a variety of configurations in $\mathrm{AdS}_{3} / \mathrm{CFT}_{2}$.

We close with a couple of discussions below:

(a) Nonspherical entangling surfaces We stress that (36) should hold only for spherical entangling surfaces, which includes all examples discussed in this paper so far. The backreaction in (35) becomes highly nontrivial when working with other geometries. For example, in $\mathrm{AdS}_{3} / \mathrm{CFT}_{2}$, if we bipartition the space into the union of two intervals and its complement, the entangling surface is no longer a sphere (two points in this dimension). Because we are working with the vacuum, we know that $\mathcal{E}_{A}=S_{1 / 2}\left(\rho_{A}\right)$. As the crossratio is varied, the proportionality between the negativity and the area of the entanglement wedge cross section changes (see Fig. 11).

(b) Bit threads We recall that the entanglement wedge is the bulk region corresponding to the reduced density

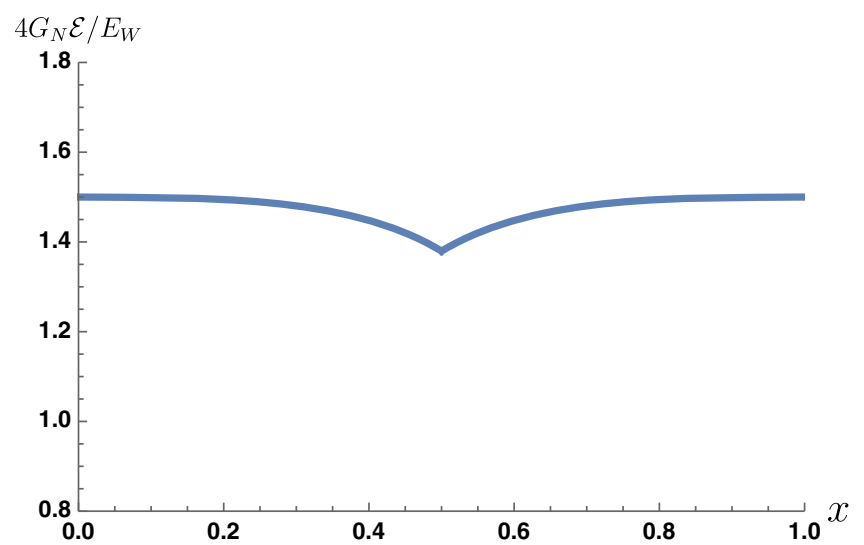

FIG. 11. As we vary the cross-ratio, $x$, the proportionality between the negativity and area of the entanglement wedge cross section, $\mathcal{E} / S=S_{1 / 2} / S$, is perturbed from the value of $3 / 2$ known for spherical entangling surfaces. Here, the Rényi entropy $S_{1 / 2}$ was computed using the series expansion derived in Ref. [68].

matrix on the boundary. We can formulate the relation between the negativity and the entanglement wedge from the perspective of bit threads [74] by stating that the negativity between two boundary regions $A$ and $B$ is proportional to the maximum number of bit threads connecting the two regions through the bulk dual of $\rho_{A B}$. The maximization procedure is taken over all possible bit thread configurations. Unlike the case of entanglement entropy, the bit threads can no longer end on horizons. Another simplifying aspect in the case of negativity is that the bit threads do not have to be directed. To account for nonspherically shaped entangling surfaces and Rényi entropies, it would be interesting to formulate bit threads in a language that could account for backreaction.

A similar picture can be made when considering entanglement of purification. This time, the horizons represent the larger boundary Hilbert space needed to purify $\rho_{A B}$. In the effective bulk, there are no more horizons, so minimizing the maximum number of bit threads connecting the purified spaces of $A$ and $B$ is again proportional to the entanglement wedge cross section. If we are forced to use the horizons as the purifying Hilbert space, then the conjecture from Ref. [37] would be proven, though this is a highly nontrivial assumption.

Interestingly, explicit bit thread configurations in the entanglement wedge have been constructed in Ref. [75]. There, the bit threads were interpreted as the maximum number of Bell pairs that can be distilled from $\rho_{A B}$. This interpretation is extremely similar to that of logarithmic negativity, which provides a bound on the distillable entanglement of mixed states [54].

(c) Covariant conjecture A natural covariant generalization may be considered in a way similar to the HRT formula. Here, we would need to find the proper 
analytic continuation of extremal cosmic branes in the entanglement wedge.

It would be fascinating to explore these generalizations quantitatively in order to better understand the connection between negativity and entanglement wedge cross sections.

\section{ACKNOWLEDGMENTS}

We thank Tom Faulkner, Ian MacCormack, Umang Mehta, Masahiro Nozaki, Hassan Shapourian, Tadashi Takayanagi, Mao Tian Tan, and Xueda Wen for useful discussions. S. R. is supported by a Simons Investigator Grant from the Simons Foundation.

\section{APPENDIX A: OPERATOR-ALGEBRA QEC}

It was shown in Ref. [8] that subsystem error-correcting codes are not general enough to serve as a formal framework for holography. This is because in subsystem error-correcting codes the area operator is proportional to the identity and is hence trivial. In AdS/CFT, the area operator cannot be trivial. To remedy this, the codes are generalized such that they require only the recovery of a subalgebra of the observables on $\mathcal{H}_{\text {code }}$. The subalgebras of interest are von Neumann algebras. For finite-dimensional Hilbert spaces, the von Neumann algebra $M$ on $\mathcal{H}_{\text {code }}$ decomposes $\mathcal{H}_{\text {code }}$ as

$$
\mathcal{H}_{\text {code }}=\oplus_{\alpha}\left(\mathcal{H}_{a_{\alpha}} \otimes \mathcal{H}_{\bar{a}_{\alpha}}\right)
$$

such that all of the operators $\tilde{O} \in M$ are block diagonal in $\alpha$ and are decomposed in each block as $\tilde{O}_{a_{\alpha}} \otimes I_{\bar{a}_{\alpha}}$. The orthonormal basis of states can be decomposed as

$$
\begin{aligned}
|\widetilde{\alpha, i j}\rangle & \equiv|\widetilde{\alpha, i}\rangle \otimes|\widetilde{\alpha, j}\rangle, \\
\tilde{\rho}_{\alpha \alpha} & =|\widetilde{\alpha, i j}\rangle\langle\widetilde{\alpha, i j}| .
\end{aligned}
$$

Von Neumann entropy can now be decomposed into a classical Shannon entropy and entanglement entropies weighted by the block probabilities,

$$
\begin{aligned}
p_{\alpha} \tilde{\rho}_{a_{\alpha}} & \equiv \operatorname{Tr}_{\bar{a}_{\alpha}} \tilde{\rho}_{\alpha \alpha}, \\
S(\tilde{\rho}, M) & \equiv-\sum_{\alpha} p_{\alpha} \ln p_{\alpha}+\sum_{\alpha} p_{\alpha} S\left(\tilde{\rho}_{a_{\alpha}}\right) .
\end{aligned}
$$

Analogously to the section on conventional error correction, the code space is spanned by

$$
\begin{aligned}
|\widetilde{\alpha, i j}\rangle & =U_{A}\left(|\alpha, i\rangle_{A_{1}^{\alpha}} \otimes\left|\chi_{\alpha, j}\right\rangle_{A_{2}^{\alpha}, \bar{A}}\right) \\
& =U_{\bar{A}}\left(|\alpha, j\rangle_{\bar{A}_{1}^{\alpha}} \otimes\left|\chi_{\alpha, i}\right\rangle_{\bar{A}_{2}^{\alpha}, A}\right)
\end{aligned}
$$

and thus simplifies to

$$
|\widetilde{\alpha, i j}\rangle=U_{A} U_{\bar{A}}\left(|\alpha, i\rangle_{A_{1}^{\alpha}}|\alpha, j\rangle_{\bar{A}_{1}^{\alpha}} \otimes\left|\chi_{\alpha}\right\rangle_{A_{2}^{\alpha}, \bar{A}_{2}^{\alpha}}\right) .
$$

The resulting density matrices are

$$
\begin{aligned}
& \tilde{\rho}_{A}=U_{A}\left(\oplus_{\alpha}\left(p_{\alpha} \rho_{A_{1}^{\alpha}} \otimes \chi_{A_{2}^{\alpha}}\right)\right) U_{A}^{\dagger}, \\
& \tilde{\rho}_{\bar{A}}=U_{\bar{A}}\left(\oplus_{\alpha}\left(p_{\alpha} \rho_{\bar{A}_{1}^{\alpha}} \otimes \chi_{\bar{A}_{2}^{\alpha}}\right)\right) U_{\bar{A}}^{\dagger} .
\end{aligned}
$$

A new area operator is then defined,

$$
\mathcal{L}_{A} \equiv \oplus_{\alpha} S\left(\chi_{A_{2}^{\alpha}}\right) I_{a_{\alpha} \bar{a}_{\alpha}},
$$

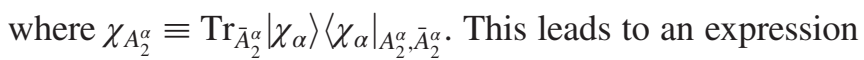
for the entropies,

$$
\begin{aligned}
& S\left(\tilde{\rho}_{A}\right)=\operatorname{Tr} \tilde{\rho} \mathcal{L}_{A}+S(\tilde{\rho}, M), \\
& S\left(\tilde{\rho}_{\bar{A}}\right)=\operatorname{Tr} \tilde{\rho} \mathcal{L}_{A}+S\left(\tilde{\rho}, M^{\prime}\right),
\end{aligned}
$$

where $M^{\prime}$ is the commutant of $M$, i.e., all operators on $\mathcal{H}_{\text {code }}$ that commute with all operators in $M$. Happily, the area operator is no longer trivial.

\section{Negativity}

Repeating an exercise similar to that in Sec. II B, we perform Schmidt decompositions on the entangling resources,

$$
\begin{aligned}
\left|\chi_{\alpha, j}\right\rangle_{A_{2}^{\alpha}, \bar{A}} & =\sum_{a} \sqrt{P_{a, \alpha}}|a\rangle_{A_{2}^{\alpha}}|a\rangle_{\bar{A}}, \\
\left|\chi_{\beta, j}\right\rangle_{\bar{A}_{2}^{\beta}, A} & =\sum_{b} \sqrt{P_{b, \beta}}|b\rangle_{\bar{A}_{2}^{\beta}}|b\rangle_{A},
\end{aligned}
$$

which lead us to the (equal) negativities of $A$ and $\bar{A}$,

$$
\begin{aligned}
\mathcal{E}\left(\rho_{A}\right) & =\sum_{\alpha} p_{\alpha}\left(\sum_{a} \sqrt{P_{a, \alpha}}\right)^{2}+\mathcal{E}(\tilde{\rho}, M) \\
& =\operatorname{Tr} \tilde{\rho}\left(\sum_{a} \sqrt{P_{a, \alpha}}\right)^{2}+\mathcal{E}(\tilde{\rho}, M), \\
\mathcal{E}\left(\rho_{\bar{A}}\right) & =\sum_{\alpha} p_{\beta}\left(\sum_{b} \sqrt{P_{b, \beta}}\right)^{2}+\mathcal{E}(\tilde{\rho}, M) \\
& =\operatorname{Tr} \tilde{\rho}\left(\sum_{b} \sqrt{P_{b, \beta}}\right)^{2}+\mathcal{E}(\tilde{\rho}, M) .
\end{aligned}
$$

If the $\chi_{A_{2}^{\alpha}}$ 's are maximally mixed, then we maintain a generalized area formula,

$$
\mathcal{E}\left(\rho_{\bar{A}}\right)=\mathcal{E}\left(\rho_{A}\right)=\left\langle\mathcal{L}_{A}\right\rangle+\mathcal{E}(\tilde{\rho}, M) .
$$

This representation of negativity shows a weighted sum of the negativities of each block. This feature appears more broadly for density matrices of block diagonal form, not just for error-correcting codes. When the density matrix is of block diagonal form, 
$\rho_{\mathcal{A} \mathcal{A}^{\prime}}=\left(\begin{array}{cccc}p_{1} \rho_{\mathcal{A}_{1} \mathcal{A}_{1}^{\prime}} & 0 & \cdots & 0 \\ 0 & p_{2} \rho_{\mathcal{A}_{2} \mathcal{A}_{2}^{\prime}} & \cdots & 0 \\ \vdots & \vdots & & \vdots \\ 0 & 0 & \cdots & p_{m} \rho_{\mathcal{A}_{m} \mathcal{A}_{m}^{\prime}}\end{array}\right)$,

its partial transpose is

$\rho_{\mathcal{A} \mathcal{A}^{\prime}}^{T_{\mathcal{A}}}=\left(\begin{array}{cccc}p_{1} \rho_{\mathcal{A}_{1} \mathcal{A}_{1}^{\prime}}^{T_{\mathcal{A}}} & 0 & \cdots & 0 \\ 0 & p_{2} \rho_{\mathcal{A}_{2} \mathcal{A}_{2}^{\prime}}^{T_{\mathcal{A}}} & \cdots & 0 \\ \vdots & \vdots & & \vdots \\ 0 & 0 & \cdots & p_{m} \rho_{\mathcal{A}_{m} \mathcal{A}_{m}^{\prime}}^{T_{\mathcal{A}}}\end{array}\right)$

This simply leads us to the negativity

$$
\mathcal{E}\left(\rho_{\mathcal{A} \mathcal{A}^{\prime}}\right)=\sum_{i} p_{i} \mathcal{E}\left(\rho_{\mathcal{A}_{i} \mathcal{A}_{i}^{\prime}}\right)
$$

It is interesting to note that, unlike von Neumann entropy, the negativity does not contain a classical Shannon-like term representing the entropy between the sectors.

\section{APPENDIX B: OPTIONAL REMOVAL OF HORIZON TENSORS}

We explain how to remove an additional layer of our tensor network and when this procedure is valid. We introduce the decompositions of the Hilbert spaces (both bulk and boundary) as follows. The boundary Hilbert space is decomposed into two parts, $\mathcal{H}_{A B} \otimes \mathcal{H}_{C}$. As for the bulk, there are d.o.f. defined for the dangling points in the tensor network, as well as those living on bonds. The latter d.o.f. correspond to $|\chi\rangle$ in the generic descriptions of subsystem QEC with complementary recovery. As for the "dangling" d.o.f., we decompose them as $\mathcal{H}_{b_{A B}} \otimes \mathcal{H}_{b_{C}}$, where $b_{A B}$ represents the dangling Hilbert space on the entanglement wedge of $A B$, whereas $b_{C}$ lives on the entanglement wedge of $C$. We further decompose $b_{A B}$ into $\tilde{b}_{A B}$ and $b_{h}$, where $b_{h}$ represents dangling d.o.f. living on the "horizon"; namely, we identify by the greedy algorithm the minimal surface which cuts bonds connecting the entanglement wedge of $A B$ and $C . b_{h}$ are defined just inside of the horizon. We have a similar decomposition of the Hilbert space associated to the bulk link d.o.f. $\mathcal{H}_{l_{C}} \otimes \mathcal{H}_{l_{A B}} \otimes \mathcal{H}_{l_{e}}$, where $l_{A B}$ represents the link Hilbert space on the entanglement wedge of $A B$, whereas $l_{C}$ lives on the entanglement wedge of $C$, and finally, $l_{e}$ represents all links cut by the minimal surface.

In Sec. II C 2, We are interested in the reduced density matrix $\rho_{A B}$ on $\mathcal{H}_{A B}$ (or $\tilde{\rho}_{A B}$ in the notation we used in QEC section). This is obtained from the total density matrix $\rho_{A B C}$ on $\mathcal{H}_{A B C}$ by taking partial trace

$$
\rho_{A B}=\operatorname{Tr}_{C} \rho_{A B C}
$$

(For our situation, $\rho_{A B C}$ is pure.)

By using the isometry $W$ from $\mathcal{H}_{b_{C}} \otimes \mathcal{H}_{l_{e}}$ to $\mathcal{H}_{C}$, the reduced density matrix can be written as

$$
\rho_{A B}=\operatorname{Tr}_{C} W \rho_{A B C} W^{\dagger}=\operatorname{Tr}_{b_{C}, l_{e}} \rho_{A B, b_{C} l_{e}},
$$

where $\rho_{A B, b_{C}, l_{e}}$ is the result of the isometric map. The d.o.f. $b_{h}$ are straightforward to trace over because $\rho_{A B, b_{C}, l_{e}}$ is a separable state,

$$
\rho_{A B, b_{C}, l_{e}}=\sum_{i} p_{i} \rho_{A B, l_{e}}^{i} \otimes \rho_{b_{C}}^{i} .
$$

For example, if $\rho_{A B, b_{C}, l_{e}}$ is pure,

$$
\rho_{A B, b_{C}, l_{e}}=\left|\psi_{A B, b_{C}, l_{e}}\right\rangle\left\langle\psi_{A B, b_{C}, l_{e}}\right|
$$

with $\left|\psi_{A B, b_{C}, l_{e}}\right\rangle=\left|\psi_{A B, l_{e}}\right\rangle \otimes\left|\psi_{b_{C}}\right\rangle$, then $\rho_{A B}$ is given by

$$
\rho_{A B}=\operatorname{Tr}_{l_{e}}\left|\psi_{A B, l_{e}}\right\rangle\left\langle\psi_{A B, l_{e}}\right| .
$$

For our purpose, we want to write $\rho_{A B}$ using the d.o.f. living on $b_{h}$. We find this is possible under a certain condition, but not in general. To state the condition, we focus on (for simplicity) the case where both the bulk state that we feed in to the QEC and the boundary states are pure, and given by $|\psi\rangle_{b}$ and $|\psi\rangle_{A B C}$, respectively.

Recall that the tensor network (QEC) acts as an isometry from the (dangling) bulk to the boundary; i.e., there is an isometry relating $\left|\psi_{b}\right\rangle$ and $\left|\psi_{A B C}\right\rangle$. This means, in particular, if we Schmidt decompose $\left|\psi_{b}\right\rangle$ as

$$
\left|\psi_{b}\right\rangle=\sum_{i} c_{i}\left|\psi_{\tilde{b}_{A B}, b_{C}}^{i}\right\rangle \otimes\left|\psi_{b_{h}}^{i}\right\rangle
$$

each term in the decomposition is mapped to a corresponding state $\left|\psi_{A B C}^{i}\right\rangle$, and hence we have a decomposition,

$$
\left|\psi_{A B C}\right\rangle=\sum_{i} c_{i}\left|\psi_{A B C}^{i}\right\rangle .
$$

We engineer the state

$$
\left|\phi_{A B C, b_{h}}\right\rangle=\sum_{i} c_{i}\left|\psi_{A B C}^{i}\right\rangle \otimes\left|\psi_{b_{h}}^{i}\right\rangle
$$

and assume it is a product state,

$$
\left|\phi_{A B C, b_{h}}\right\rangle=\left|\psi_{A B C}\right\rangle \otimes\left|\psi_{b_{h}}\right\rangle .
$$

This is our condition for removing the "horizon layer" of the tensor network. Then, in this case, $\rho_{A B C}$ can be represented as a partial trace over $b_{h}$,

$$
\rho_{A B C}=\operatorname{Tr}_{b_{h}}|\phi\rangle\left\langle\left.\phi\right|_{A B C, b_{h}},\right.
$$


where $\left|\phi_{A B C, b_{h}}\right\rangle$ is given by

$$
\left|\phi_{A B C, b_{h}}\right\rangle=|\psi\rangle_{A B C} \otimes|\psi\rangle_{b_{h}} .
$$

Now, for the case of this "special class" of bulk states, the tensor removal procedure by isometry can be repeated, to reach (B5), but since $\rho_{A B C}$ can now be written with a partial trace over $b_{h}$ of the engineered state $|\phi\rangle_{A B C, b_{h}}$,

$$
\rho_{A B}=\operatorname{Tr}_{b_{h}} \operatorname{Tr}_{l_{e}}|\phi\rangle\left\langle\left.\phi\right|_{A B, l_{e}, b_{h}},\right.
$$

where $\left|\phi_{A B, l_{e}, b_{h}}\right\rangle$ is obtained from $\left|\phi_{A B C, b_{h}}\right\rangle$ by applying the isometry $W$.

Applying an additional isometry, we can now remove d.o.f. in $b_{h}$ and $l_{e}$. After taking these partial traces, we are now left with the description of $\rho_{A B}$ as the effective bulk state fed into the (remaining) effective tensor network. In particular, the part of the effective tensor network that previously connected $\tilde{b}_{A B}$ and $b_{h}$ can now be regarded as a horizon in the sense that we described before around Fig. 7; after removing $b_{h}$, these links are now dangling.
[1] J. Maldacena, Int. J. Theor. Phys. 38, 1113 (1999).

[2] E. Witten, Adv. Theor. Math. Phys. 2, 253 (1998).

[3] S. S. Gubser, I. R. Klebanov, and A. M. Polyakov, Phys. Lett. B 428, 105 (1998).

[4] T. Nishioka, S. Ryu, and T. Takayanagi, J. Phys. A 42, 504008 (2009).

[5] T. Takayanagiand M. Rangamani, Holographic Entanglement Entropy, in Lecture Notes in Physics, edited by $\mathrm{M}$. Rangamani and T. Takayanagi (Springer Verlag, Berlin, 2017), Vol. 931.

[6] T. Nishioka, Rev. Mod. Phys. 90, 035007 (2018).

[7] D. Harlow, Rev. Mod. Phys. 88, 015002 (2016).

[8] D. Harlow, Commun. Math. Phys. 354, 865 (2017).

[9] A. Almheiri, X. Dong, and D. Harlow, J. High Energy Phys. 04 (2015) 163.

[10] F. Pastawski, B. Yoshida, D. Harlow, and J. Preskill, J. High Energy Phys. 06 (2015) 149.

[11] S. Ryu and T. Takayanagi, Phys. Rev. Lett. 96, 181602 (2006).

[12] S. Ryu and T. Takayanagi, J. High Energy Phys. 8 (2006) 045.

[13] T. Faulkner, A. Lewkowycz, and J. Maldacena, J. High Energy Phys. 13 (2013) 74.

[14] X. Dong, J. High Energy Phys. 01 (2014) 44.

[15] P. Hayden, S. Nezami, X.-L. Qi, N. Thomas, M. Walter, and Z. Yang, J. High Energy Phys. 11 (2016) 009.

[16] P. Calabrese, J. Cardy, and E. Tonni, Phys. Rev. Lett. 109, 130502 (2012).

[17] P. Calabrese, J. Cardy, and E. Tonni, J. Stat. Mech. (2013) P02008.

[18] P. Calabrese, L. Tagliacozzo, and E. Tonni, J. Stat. Mech. (2013), P05002.

[19] V. Alba, J. Stat. Mech. (2013), P05013.

[20] C.-M. Chung, V. Alba, L. Bonnes, P. Chen, and A. M. Läuchli, Phys. Rev. B 90, 064401 (2014).

[21] P. Ruggiero, V. Alba, and P. Calabrese, Phys. Rev. B 94, 035152 (2016).

[22] P. Ruggiero, V. Alba, and P. Calabrese, Phys. Rev. B 94, 195121 (2016).

[23] O. Blondeau-Fournier, O. A. Castro-Alvaredo, and B. Doyon, J. Phys. A 49, 125401 (2016).

[24] V. Eisler and Z. Zimborás, New J. Phys. 16, 123020 (2014).
[25] A. Coser, E. Tonni, and P. Calabrese, J. Stat. Mech. (2014) P12017.

[26] M. Hoogeveen and B. Doyon, Nucl. Phys. B898, 78 (2015).

[27] X. Wen, P.-Y. Chang, and S. Ryu, Phys. Rev. B 92, 075109 (2015).

[28] C. Castelnovo, Phys. Rev. A 88, 042319 (2013).

[29] Y. A. Lee and G. Vidal, Phys. Rev. A 88, 042318 (2013).

[30] X. Wen, P.-Y. Chang, and S. Ryu, J. High Energy Phys. 9 (2016) 012.

[31] X. Wen, S. Matsuura, and S. Ryu, Phys. Rev. B 93, 245140 (2016).

[32] M. Headrick, V. E. Hubeny, A. Lawrence, and M. Rangamani, J. High Energy Phys. 12 (2014) 162.

[33] D. L. Jafferis and S. J. Suh, J. High Energy Phys. 09 (2016) 068.

[34] D. L. Jafferis, A. Lewkowycz, J. Maldacena, and S. J. Suh, J. High Energy Phys. 06 (2016) 004.

[35] V. E. Hubeny and M. Rangamani, J. High Energy Phys. 6 (2012) 114.

[36] M. Headrick, V. E. Hubeny, A. Lawrence, and M. Rangamani, J. High Energy Phys. 12 (2014) 162.

[37] T. Takayanagi and K. Umemoto, Nat. Phys. 14, 573 (2018).

[38] A. Bhattacharyya, T. Takayanagi, and K. Umemoto, J. High Energy Phys. 04 (2018) 132.

[39] P. Nguyen, T. Devakul, M. G. Halbasch, M. P. Zaletel, and B. Swingle, J. High Energy Phys. 01 (2018) 98.

[40] H. Hirai, K. Tamaoka, and T. Yokoya, Prog. Theor. Exp. Phys. 2018, 063 B03 (2018).

[41] N. Bao and I. F. Halpern, J. High Energy Phys. 03 (2018) 006.

[42] R. Espíndola, A. Guijosa, and J. F. Pedraza, Eur. Phys. J. C 78, 646 (2018).

[43] N. Bao and I. F. Halpern, Phys. Rev. D 99, 046010 (2019).

[44] K. Umemoto and Y. Zhou, J. High Energy Phys. 10 (2018) 152.

[45] Y. Nomura, P. Rath, and N. Salzetta, Phys. Rev. D 98, 026010 (2018).

[46] P. Jain, V. Malvimat, S. Mondal, and G. Sengupta, Phys. Lett. B 793, 104 (2019).

[47] P. Chaturvedi, V. Malvimat, and G. Sengupta, Eur. Phys. J. C 78, 499 (2018). 
[48] P. Jain, V. Malvimat, S. Mondal, and G. Sengupta, Eur. Phys. J. Plus 133, 300 (2018).

[49] P. Chaturvedi, V. Malvimat, and G. Sengupta, J. High Energy Phys. 05 (2018) 172.

[50] P. Jain, V. Malvimat, S. Mondal, and G. Sengupta, arXiv: 1710.06138.

[51] A. Peres, Phys. Rev. Lett. 77, 1413 (1996).

[52] M. Horodecki, P. Horodecki, and R. Horodecki, Phys. Lett. A 223, 1 (1996).

[53] J. Eisert and M. B. Plenio, J. Mod. Opt. 46, 145 (1999).

[54] G. Vidal and R.F. Werner, Phys. Rev. A 65, 032314 (2002).

[55] M. B. Plenio, Phys. Rev. Lett. 95, 090503 (2005).

[56] V. E. Hubeny, M. Rangamani, and T. Takayanagi, J. High Energy Phys. 07 (2007) 062.

[57] A. May, J. High Energy Phys. 06 (2017) 118.

[58] A. C. Wall, Classical Quantum Gravity 31, 225007 (2014).

[59] We would end up with the same result for negativity if we took the partial transpose with respect to $A$ because the negativity is symmetric about bipartite states.

[60] Under certain conditions, we can optionally remove one horizon layer of tensors from the tensor network. This transfers negativity from the area term to the bulk term in (28). We discuss this technicality in Appendix B.

[61] X. Dong, Nat. Commun. 7, 12472 (2016).
[62] C. Akers and P. Rath, arXiv:1811.05171.

[63] X. Dong, D. Harlow, and D. Marolf, arXiv:1811.05382.

[64] L.-Y. Hung, R. C. Myers, M. Smolkin, and A. Yale, J. High Energy Phys. 12 (2011) 47.

[65] M. Rangamani and M. Rota, J. High Energy Phys. 10 (2014) 60.

[66] P. Calabrese, J. Cardy, and E. Tonni, J. Phys. A 48, 015006 (2015).

[67] M. Kulaxizi, A. Parnachev, and G. Policastro, J. High Energy Phys. 09 (2014) 10.

[68] T. Hartman, arXiv:1303.6955.

[69] T. Faulkner, arXiv:1303.7221.

[70] A. B. Zamolodchikov, Theor. Math. Phys. 73, 1088 (1987).

[71] M. Headrick, Phys. Rev. D 82, 126010 (2010).

[72] V. Malvimat and G. Sengupta, arXiv:1712.02288.

[73] J. Maldacena, J. High Energy Phys. 04 (2003) 021.

[74] M. Freedman and M. Headrick, Commun. Math. Phys. 352, 407 (2017).

[75] C. A. Agón, J. de Boer, and J.F. Pedraza, arXiv:1811 .08879 .

Correction: The omission of a support statement in the Acknowledgments section has been fixed. 